\title{
Coordinated Regulation of Dendrite Arborization by Epigenetic Factors CDYL and EZH2
}

\author{
Cai Qi, ${ }^{1 \star}$ Shumeng Liu, ${ }^{2 \star}$ Rui Qin, ${ }^{1 \star}$ Yu Zhang, ${ }^{2}$ Guoqiang Wang, ${ }^{1}$ Yongfeng Shang, ${ }^{2,3}$ Yun Wang,,${ }^{1,4}$ and Jing Liang ${ }^{2}$ \\ ${ }^{1}$ Neuroscience Research Institute, Department of Neurobiology, School of Basic Medical Sciences, Key Laboratory for Neuroscience, Ministry of Education/ \\ National Health and Family Planning Commission and ${ }^{2}$ Key Laboratory of Carcinogenesis and Translational Research (Ministry of Education), Department \\ of Biochemistry and Molecular Biology, Peking University Health Science Center, Beijing 100191, China, ${ }^{3}$ Tianjin Key Laboratory of Medical Epigenetics, \\ Tianjin Medical University, Tianjin 300070, China, and ${ }^{4}$ KKU-IDG/McGovern Institute for Brain Research, Peking University, Beijing 100871, China
}

Dendritic arborization is one of the key determinants of precise circuits for information processing in neurons. Unraveling the molecular mechanisms underlying dendrite morphogenesis is critical to understanding the establishment of neuronal connections. Here, using gain- and loss-of-function approaches, we defined the chromodomain protein and transcription corepressor chromodomain Y-like (CDYL) protein as a negative regulator of dendrite morphogenesis in rat/mouse hippocampal neurons both in vitro and in vivo. Overexpressing CDYL decreased, whereas knocking it down increased, the dendritic complexity of the primary cultured rat neurons. Highthroughput DNA microarray screening identified a number of CDYL downstream target genes, including the brain-derived neurotrophic factor (BDNF). Knock-down of CDYL in neuronal cells led to increased expression of BDNF, which is primarily responsible for CDYL's effects on dendrite patterns. Mechanistically, CDYL interacts with EZH2, the catalytic subunit of Polycomb Repressive Complex 2 (PRC2), directly and recruits the H3K27 methyltransferase activity to the promoter region of the BDNF gene. In doing so, CDYL and EZH2 coordinately restrict dendrite morphogenesis in an interdependent manner. Finally, we found that neural activity increased dendritic complexity through degradation of CDYL protein to unleash its inhibition on BDNF. These results link, for the first time, the epigenetic regulators $\mathrm{CDYL}$ and $\mathrm{EZH} 2$ to dendrite morphogenesis and might shed new light on our understanding of the regulation of the neurodevelopment.

\section{Introduction}

Epigenetic regulation, including DNA methylation, histone modifications, and chromatin remodeling, has been found to play an important role in the nervous system (Borrelli et al., 2008; Ma et al., 2010; Riccio, 2010; Gräff et al., 2011; Jakovcevski and Akbarian, 2012). In particular, covalently modified histone residues, either alone or in combination, create docking sites for specific "reader" proteins, which subsequently recruit transcriptional regulators to tune up or tune down the expression level of local genes. Although several histone modifiers have been implicated in various aspects of neurobiology, such as cell death, learn-

\footnotetext{
Received Aug. 26, 2013; revised Feb. 4, 2014; accepted Feb. 13, 2014.

Author contributions: C.Q., Y.W., and J.L. designed research; C.Q., S.L., and R.Q. performed research; C.Q., S.L., R.Q., Y.Z., G.-Q.W., Y.W., and J.L. analyzed data; C.Q., Y.S., Y.W., and J.L. wrote the paper.

This work was supported by the National Natural Science Foundation of China (Grants 81161120497, 30925015 , and 30830044 to Y.W. and Grants 90919045 and 31371301 to J.L.); the Ministry of Education of China (Grant NCET-09-0184 to J.L.); and the Ministry of Science and Technology of China (973 Program Grants 2011 CB504204 and 2014 CB542004 to J.L. and Y.S. and Grant 2014CB542204 to Y.W.). We thank Dr. D.M. Yin of Institute of Molecular Medicine and Genetics, Georgia Health Sciences University, for discussions and critical reading of the manuscript.

The authors declare no competing financial interests.

${ }^{*}$ C.Q., S.L., and R.Q. contributed equally to this work.

Correspondence should be addressed to either Yun Wang, Neuroscience Research Institute, Department of Neurobiology, School of Basic Medical Sciences, Key Laboratory for Neuroscience, Ministry of Education/National Health and Family Planning Commission, or Jing Liang, Key Laboratory of Carcinogenesis and Translational Research (Ministry of Education), Department of Biochemistry and Molecular Biology, Peking University Health Science Center, Beijing 100191, China, E-mail: wangy66@bjmu.edu.cn or liang_jing@bjmu.edu.cn.

DOI:10.1523/JNEUROSCI.3647-13.2014

Copyright $\odot 2014$ the authors $\quad 0270-6474 / 14 / 344494-15 \$ 15.00 / 0$
}

ing, and memory (Wu et al., 2007; Kim et al., 2008; Nott et al., 2008; Qiu and Ghosh, 2008; Guan et al., 2009; Riccio, 2010; Sawarkar and Paro, 2010; Tea et al., 2010; Jakovcevski and Akbarian, 2012), little is known about how epigenetic mechanisms contribute to dendrite morphogenesis, a process critical for the establishment of neural circuits (Jan and Jan, 2010).

Chromodomain Y-like (CDYL) protein, containing an $\mathrm{N}$-terminal chromodomain and a carboxy-terminal enoylcoenzyme A hydratase-isomerase catalytic domain, has been implicated in transcription repression (Lahn and Page, 1999; Caron et al., 2003). It has been shown that CDYL is a component of repressor complex CtBP (Shi et al., 2003) and that, under certain conditions, CDYL bridges the transcription repressor RE1silencing transcription factor (REST) and the histone H3K9 methyltransferase G9a for repression of gene transcription (Mulligan et al., 2008). The chromodomain of CDYL is believed to read histone lysine methylation (Kim et al., 2006; Vermeulen et al., 2010) and we demonstrated previously that CDYL can recognize the repressive chromatin mark $\mathrm{H} 3 \mathrm{~K} 27$ trimethylation (H3K27me3) and recruit the Polycomb Repressive Complex 2 (PRC2) to the chromatin substrate (Zhang et al., 2011). Although it is becoming clear that CDYL is a transcriptional corepressor and an important epigenetic regulator, the in vivo target genes of CDYL are poorly defined and the biological function of this protein remains to be determined.

Although homologs of CDYL have been shown to play a key role in mammalian spermatogenesis (Lahn et al., 2002), the fact 
that CDYL is widely expressed in various tissues (Lahn and Page, 1999) suggests that it might have a broad functionality. Indeed, analysis of our previous CDYL ChIP-sequencing studies (ChIPSeq; Zhang et al., 2011) by functional taxonomy and gene ontology (GO) found, unexpectedly, a significant number of CDYL target genes implicated in the development and functionality of the nervous system and a significant recurrent appearance of the REST motif in $\sim 500$ CDYL-binding sites. Because REST is a classical transcriptional repressor primarily implicated in neurological functions, we were prompted to investigate whether and how CDYL might play a role in the nervous system.

Here, we report that CDYL is a negative regulator of dendrite branching. We show that CDYL recruits the H3K27 methyltransferase activity to the brain-derived neurotrophic factor (BDNF) promoter to regulate gene transcription and, consequently, CDYL and EZH2 coordinately regulate dendrite morphogenesis. Neuronal CDYL protein is rapidly degraded during $\mathrm{KCl}$ stimulation, indicating that CDYL is an important regulator of dendrite morphogenesis in activity-dependent neuronal function.

\section{Materials and Methods}

Plasmids. Human CDYL was cloned into PBK vector and pEGFP-N1 vector to produce FLAG-tagged and C-terminal green fluorescent protein (GFP)-tagged expression plasmid, respectively. Short hairpin RNA (shRNA) plasmids specific for rat Cdyl were produced by insertion of the following sequence into pSUPER basic vector: sense: $5^{\prime}$-GATCCCCGGTACATCTCCATTCATGG-TTCAAGAGA-CCATGAATGGAGATGTACC-TTTTTA-3'; antisense: 5' -AGCTTAAAAA-GGTACATCTCC ATTCATGG-TCTCTTGAA-CCATGAATGGAGATGTACC-GGG-3' . The sequences for nonsilencing shRNA were as follows: sense: 5'-GATCCCC-GCAAATTCTCGGTGTCAAA-TTCAAGAGA-TTTGACACCGAGAATTTGC-TTTTTA-3'; antisense: 5' -AGCTTAAAAAGCAAATTCTCGGTGTCAAA-TCTCTTGAA-TTTGACACCGAGA ATTTGC-GGG-3'. To create shRNA-resistant CDYL (CDYL-R), the third nucleotide of each codon in the target sequence was mutated without changing the identity of the amino acids. For chromodomain deletion mutants, FLAG-CDYL del60 was generated by PCR. FLAG-CDYL del60-R was generated by PCR from the FLAG-CDYL-R expression plasmid. All CDYL mutants and other expression plasmids were generated using standard protocols and confirmed by sequencing. Mouse EZH2 was cloned into pcDNA3.1 vector to produce FLAG-tagged expression plasmid.

RNA interference. The following siRNAs were synthesized by Shanghai Gene Pharma. The sequences were as follows: rat EZH2 sense: GCAAAUUCUCGGUGUCAAAtt; antisense: UUUGACACCGAG AAUUUGCtt. Nonsilencing siRNA sense: UUCUCCGAACGUGUCACGUtt; antisense: ACGUGACACGUUCGGAGAAtt. Human CDYL sense: CAGAGAAUAACUCACUAAAdTdT; antisense: UUUAGUGAGUUAUUCUCUGdTdT. The following siRNAs were purchased from Sigma-Aldrich: rat BNDF\#1 (SASI_Rn01_00075927) sense: GAUAGUGGAUUUAUGUUGUdTdT; antisense: ACAACAUAAAUCCACUAUCdTdT. Rat BNDF\#2 (SASI_Rn02_00259567) sense: CUGGAACUCGCAAUGCCGAdTdT; antisense: UCGGCAUUGCGAG UUCCAGdTdT. Rat BDNF\#3 (SASI_Rn01_0075929) sense: GAGAAAGUCCCGGUAUCAAdTdT; antisense: UUGAUACCGGGACUUUCUCdTdT. Human EZH2\#1 (SASI_Hs01_00147882) sense: CAU CGAAAGAGAAAUGGAAdTdT; antisense: UUCCAUUUCUCUUUCGAUGdTdT. Human EZH2\#2 (SASI_Hs01_00147883) sense: CUAACCAUGUUUACAACUAdTdT; antisense: UAGUUGUAAACAUGG UUAGdTdT.

Cell culture and transfection. Rat C6 and human SH-SY5Y cells were maintained in DMEM supplemented with $10 \%$ fetal bovine serum (FBS). Cells were transfected with Lipofectamine RNAiMAX Transfection Reagent (Life Technologies) according to the manufacturer's instructions.

Primary neuron cultures, transfection, and reagents. Hippocampal explants isolated from embryonic day 18 rat embryos of either sex were digested with $0.25 \%$ trypsin for $30 \mathrm{~min}$ at $37^{\circ} \mathrm{C}$, followed by triturating with a pipette in plating medium (DMEM with 10\% FBS). Dissociated neurons were plated onto $35 \mathrm{~mm}$ dishes coated with poly-D-lysine (Sigma Aldrich) at a density of $5 \times 10^{5}$ cells per dish. After culturing for $4 \mathrm{~h}$, the medium was changed to neurobasal medium supplemented with 2\% B27 and 0.5 mm GlutaMAX-I (Life Technologies). To test the role of CDYL in dendritic branching, neurons were cotransfected with the DNA of interest and pEGFP-N1 at day 8 in vitro (DIV). Cells were fixed with $4 \%$ paraformaldehyde and subjected to analysis of dendritic branching $3 \mathrm{~d}$ after transfection. Lipofectamine 2000 (Life Technologies) was used for neuronal transfection following the instructions provided by the manufacturer. To address the effects of the recombinant protein TrkB-Fc on dendritic numbers, TrkB-Fc $(0.33 \mu \mathrm{g} / \mathrm{ml}$; R\&D Systems $)$ was added to neurons transfected with shRNAs $12 \mathrm{~h}$ after transfection and the neurons were cultured for another $3 \mathrm{~d}$ before analyzing the dendritic complexity. For the RNA interference experiment, EZH2 siRNA, BDNF siRNA, or control siRNA was transfected together with pEGFP-N1. For rescue experiments, the indicated constructs were transfected together with pEGFP-N1. For KCl-induced morphological analysis of dendritic complexity, $16 \mathrm{~mm} \mathrm{KCl}$ was added into the medium $12 \mathrm{~h}$ after transfection and images were collected $2 \mathrm{~d}$ later. Two proteasome inhibitors were used in the protein degradation experiments: MG132 (C2211; Sigma-Aldrich) and clasto-lactacystin $\beta$-lactone (L7035; Sigma-Aldrich).

In utero electroporation. Female ICR mice were checked for vaginal plugs. Electroporation was performed at embryonic day 14.5 (E14.5). Mice embryos were exposed in the uterus and $1 \mu \mathrm{l}$ of DNA solution was injected into the lateral ventricle through the uterus wall, followed by electroporation. Plasmids were prepared in Milli-Q water at a concentration of $1 \mu \mathrm{g} / \mu \mathrm{l}$ for pEGFP-N1, $3 \mu \mathrm{g} / \mu \mathrm{l}$ for shRNAs, and 6 $\mu \mathrm{g} / \mu \mathrm{l}$ for CDYL-R or CDYL del60-R. Electric pulses of $36 \mathrm{~V}$ were generated with an ElectroSquireportator T830 (BTX) and applied to the cerebral wall for $50 \mathrm{~ms}$ each for a total of five pulses at an interval of $950 \mathrm{~ms}$. The numbers of mice used in control, CDYL shRNA, CDYL shRNA+CDYL-R, and CDYLshRNA+CDYLdel60-R groups were 14, 11,12 , and 7 , respectively.

Pull-down assays. Purified baculovirus-expressed FLAG-CDYL proteins (Zhang et al., 2011) were incubated for $4 \mathrm{~h}$ with anti-FLAG M2 affinity beads (Sigma-Aldrich) and the beads were washed five times with PBS before they were mixed with rat brain lysates in the binding buffer containing $0.1 \%$ Nonidet P-40 and $300 \mathrm{~mm} \mathrm{NaCl}$ overnight. For the control reactions, anti-FLAG M2 affinity beads without bound proteins were incubated with rat brain lysates. After the incubation, the beads were washed five times again with the binding buffer and bound proteins were eluted with FLAG peptide and subjected to Western blot analysis using anti-EZH2 antibodies.

Lentivirus infection. Rat cortical neurons were dissociated at E18. After $3 \mathrm{~d}$ of culture in vitro, neurons were infected with lentivirus containing nonsilencing shRNA or CDYL shRNA (Shanghai GeneChem) with a multiplicity of infection of 1 . Seven days after transfection, neurons were collected and either RNA or protein was extracted for quantitative realtime RT-PCR or Western blotting, respectively. The infection efficiency was confirmed by the expression of green fluorescent protein under microscopy.

Western blotting and ChIP. Western blotting and ChIP experiments were performed according to the protocols described previously (Wu et al., 2005; Li et al., 2009; Wang et al., 2009). Commercial antibodies used were CDYL (catalog \#5188; Abcam), EZH2 (catalog \#612667; BD Biosciences), FLAG (Sigma-Aldrich), ACTIN (Sigma-Aldrich), REST (catalog \#25398; Santa Cruz Biotechnology), H3K9me2 (catalog \#8898; Abcam), H3K9me3 (catalog \#05-1242; Millipore), H3K27me3 (catalog \#07-449; Millipore), BDNF (catalog \#2960-S; Epitomics), pan acetyl lysine (cata$\log \# 101$; PTM Biolab), and histone H3 (catalog \#1791; Abcam). A CDYL antibody that we generated previously (peptide antigen: KQKESTLTRTNRTSPNN; B\&M) was used for Western blot with rat cell lysates. The primers used for ChIP assay were as follows: ChIP-h-BDNF forward: TTTACAGCGGGGCCAAGAA; ChIP-h-BDNF reverse: TGTGTAATCCGGGCGATAGG.

$m R N A$ profiling. Total mRNA was extracted from prepared pairs of cortical cultures infected with lentivirus containing nonsilencing or 
CDYL shRNA. Microarray analysis was performed using Affymetrix rat genome 2302.0 array chips at CapitalBio. Differentially expressed genes were identified through the SAM (Tusher et al., 2001) package in $\mathrm{R}$ software after normalization with RMA (http://rmaexpress.bmbolstad. com). The annotated genes with transcript levels that were upregulated or downregulated by the CDYL shRNA by $>2$-fold or $<0.5$-fold, respectively, were considered to be significantly regulated by CDYL. Gene-ontology analysis against these two differentially regulated gene sets was performed using DAVID tools (Huang da et al., 2009). Statistical analysis and plotting were performed with $\mathrm{R}$ software (http://www.R-project.org).

Analysis of neuronal morphology. Morphology of entire neurons was shown by the expression of pEGFP-N1 constructs. Dissociated neurons grown at low density were used to determine morphological characteristics. Neurons transfected with DNA were selected by GFP expression and were photographed at $20 \times$ magnification using an Olympus fluorescent microscope. For analysis of total dendritic branch length, all arbors and their branches were traced and their length was measured using Image Pro Plus (Media Cybernetics). The experimental group was normalized to the control group, which was set to $100 \%$ for convenience. For Sholl analysis, concentric circles with $15 \mu \mathrm{m}$ (for cultured neurons) or $5 \mu \mathrm{m}$ (for in utero electroporation) differences in diameter were drawn around the cell body and the number of dendrites crossing each circle was counted manually (Sholl, 1953; Jaworski et al., 2005).

Statistical analysis. All data are represented as the mean \pm SEM. Comparisons between two groups were made using Student's unpaired twotailed $t$ tests. Comparisons among three or more groups were made using one-way ANOVA analyses followed by Bonferroni's multiplecomparisons test. Data marked with asterisks in the figures are significantly different from control as follows: ${ }^{\star} p<0.05,{ }^{* *} p<0.01$, ${ }^{* * *} p<$ 0.001 .

\section{Results \\ CDYL restricts dendritic morphogenesis through epigenetic mechanisms}

To understand what role, if any, CDYL might play in the nervous system, we first examined the regional and developmental expression pattern of CDYL in rat brain. CDYL is broadly expressed in the CNS (Fig. 1A), a result consistent with a previous study (Lahn and Page, 1999). The protein level of CDYL decreased during the maturation of the brain, suggesting that CDYL might play a negative role in neural development (Fig. 1B). We next examined the effect of gain-of-function and loss-of-function of CDYL on the morphology of neurons. In gain-of-function experiments, overexpression of full-length CDYL in cultured rat hippocampal neurons led to a significant decrease of dendritic branching and the total dendritic length compared with the control group (Fig. 1C-E). In contrast, knock-down of CDYL by shRNA (Fig. $1 F$ ) led to an increase of the dendritic branching and total dendritic length (Fig. $1 \mathrm{H}-J$ ). To exclude the possible offtarget effects of the shRNA system, rescuing experiments were performed with CDYL-R, a construct that was derived from human CDYL by silent mutation and therefore resistant to the shRNA (Fig. 1G). Expression of CDYL-R restored the dendritic morphology affected by CDYL shRNA, indicating the specific effects of CDYL shRNA (Fig. $1 H-J$ ).

Furthermore, loss-of-function experiments were performed by in utero electroporation (Saito and Nakatsuji, 2001). Specifically, CDYL shRNA or nonsilencing shRNA together with a GFP construct was transfected to mice progenitor cells at E14.5 via electroporation. At postnatal day 15 (P15), the morphology of CDYL shRNA-transfected hippocampal neurons was compared with that of nonsilencing shRNA-transfected neurons. Consistent with the in vitro results, dendritic complexity of CDYL shRNA-transfected neurons increased significantly relative to that of the control cells (Fig. 2A-E). Furthermore, rescuing ex- periments in which CDYL-R was cotransfected with CDYL shRNA to mice progenitor cells at E14.5 via electroporation completely restored the normal dendrite morphology of transfected hippocampal neurons at P15 (Fig. 2A-E). Moreover, further analysis revealed that CDYL affected the basal dendrites more dramatically than it did the apical dendrites (Fig. $2 F-G$ ). These data indicated that CDYL restricts the dendritic complexity of hippocampal neurons in vivo.

We also investigated whether the effects of CDYL depend on its chromatin-binding activity. We constructed a CDYL deletion mutant in which the chromodomain (the $\mathrm{N}$-terminal sequence of $60 \mathrm{aa})$ was removed (CDYL del60). Unlike that of wild-type CDYL, overexpression of CDYL del60 in cultured hippocampal neurons did not affect dendritic branching (Fig. 1C-E), nor could it rescue the effect of CDYL shRNA on dendritic complexity either in vitro (Fig. $1 H-J$ ) or in vivo (Fig. $2 A-G$ ), suggesting that CDYL influences dendrite morphogenesis by means of its association with chromatin histones.

\section{Genome-wide identification of CDYL target genes in cortical neurons}

In light of our observation that CDYL inhibits dendritic branching by virtue of its chromatin association and based on the general belief that CDYL is a transcriptional repressor ( $\mathrm{Li}$ et al., 2007; Zhang et al., 2011), we reasoned that CDYL might regulate dendrite morphogenesis through transcription repression of its downstream target genes. We performed genome-wide microarray analysis in cultured cortical neurons infected with lentivirus of CDYL-silencing or nonsilencing shRNA to identify the target genes of CDYL. Total RNA was extracted $7 \mathrm{~d}$ after lentiviral infection and microarray analysis was performed using Affymetrix rat genome 2302.0 array chips. Of 28,000 rat genes tested, 333 were found to be upregulated and 273 were found to be downregulated in CDYLdeficient neurons. Because CDYL acts as a transcriptional repressor, it is more likely that the upregulated genes represent the direct targets of CDYL. GO analysis showed that most of the upregulated genes in CDYL-depleted neurons are implicated in neurological functions, including synaptic transmission, response to various stimuli, and neuron differentiation (Fig. 3A). Quantitative real-time RT-PCR analysis of the representative genes from each of the six most upregulated functional clusters confirmed the microarray results (Fig. $3 B$ ).

Remarkably, BDNF, a key regulator of neural circuit development (Park and Poo, 2013), was identified among the genes that were upregulated in CDYL-deficient neurons (Fig. 3B). BDNF belongs to a family of secreted neurotrophins including nerve growth factor (NGF), neurotrophin 3 (NT3) and neurotrophin 4 (NT4), which act to promote the survival and growth of various neurons during development (Thoenen, 1991; Lu et al., 2005; Jan and Jan, 2010; Park and Poo, 2013). BDNF has been characterized as a critical factor in promoting dendritic morphogenesis in various types of neurons (Jan and Jan, 2010; Park and Poo, 2013). Quantitative real-time RTPCR and Western blot analysis confirmed the upregulation of BDNF in cortical neurons infected with CDYL-shRNA lentiviruses (Fig. 3B).

\section{BDNF is required for the effects of CDYL on dendritic branching}

We next examined whether BDNF is the major mediator of the effects of CDYL on dendritic branching. Treating CDYLtransfected hippocampal neurons with $25 \mathrm{ng} / \mathrm{ml}$ BDNF for $3 \mathrm{~d}$ 
A

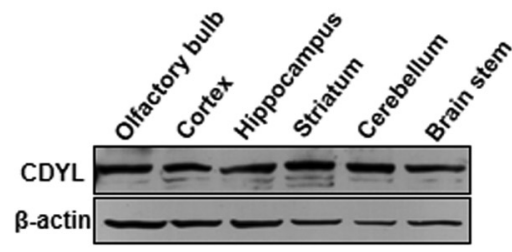

B

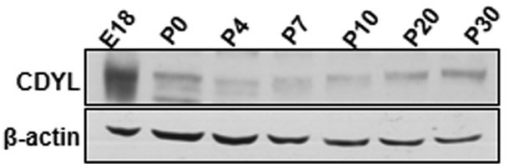

C
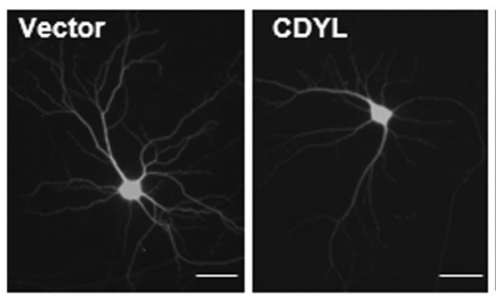

$\mathbf{F}$

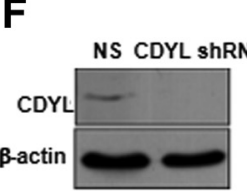

H

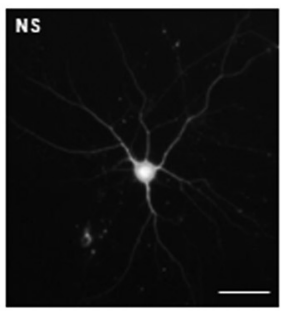

I

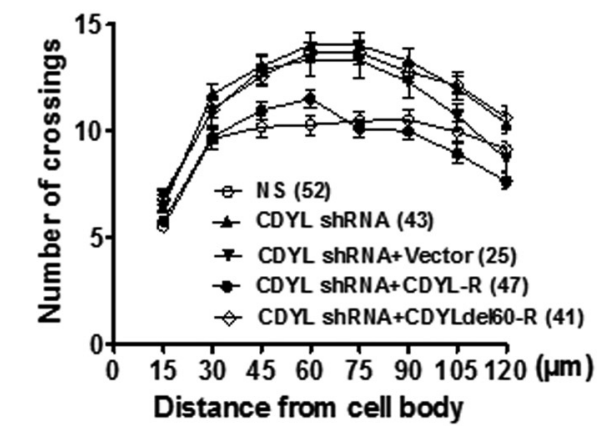

G

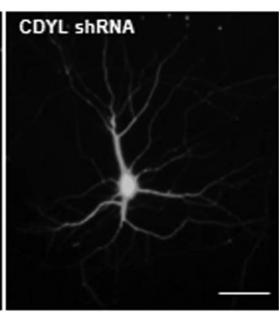

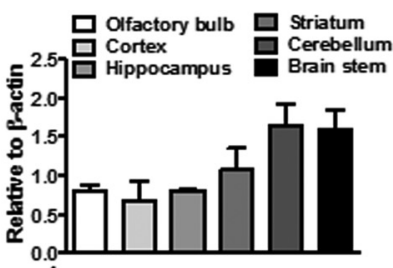

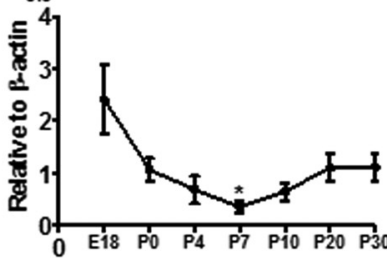

D

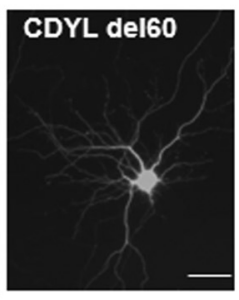

Amino acid: $G \quad T \quad S \quad P \quad F \quad M$

WT CDYL: GGT ACA TCT CCA TTC ATG

CDYL-R: $\quad$ GGT ACI TCC CCA TTC ATG

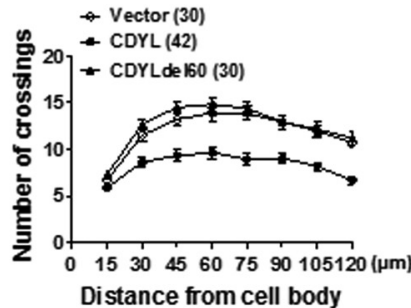

E

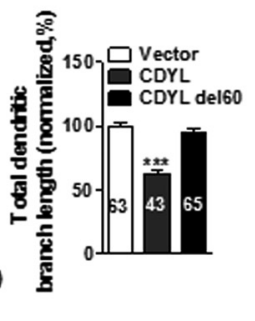

ShRNA resistant FLAG-CDYL
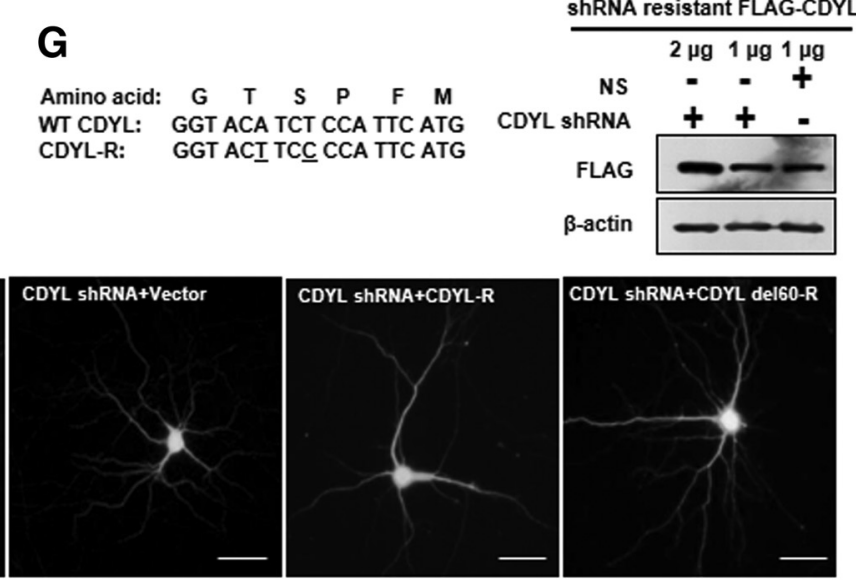

J

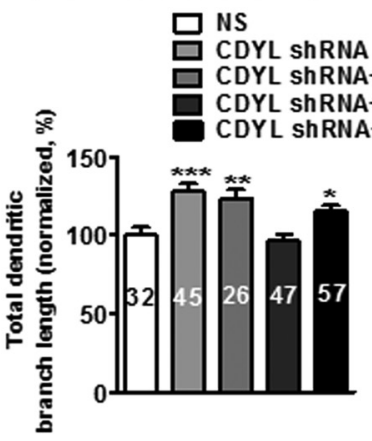

Figure 1. CDYL negatively regulates dendritic branching. $A$, Western blot analysis of CDYL in different brain areas (left). The results showed that CDYL is broadly expressed in rat brain. The blot was quantified using one-way ANOVA with Bonferroni's multiple-comparisons test (right). $\boldsymbol{B}$, Protein lysates were prepared from rat hippocampus at the indicated developmental stages (E18 to P30). Western blot analysis showed that the level of CDYL decreased as the brain matures (left). Quantification was done by normalizing the level of CDYL to that of $\beta$-actin (right). ${ }^{*} p<0.05, t$ test. C, Representative images of hippocampal neurons transfected with PBK vector, FLAG-CDYL, or FLAG-CDYL del60 at DIV 8 for $3 \mathrm{~d}$. Cells were cotransfected with GFP to visualize the morphology of the transfected neurons. Scale bar, $50 \mu \mathrm{m}$. D, Sholl analysis of hippocampal neurons transfected with the indicated constructs as shown in $\boldsymbol{C}$. The number labeled in the graph indicates the number of neurons analyzed. $\boldsymbol{E}$, Quantification of the total dendritic branch length of hippocampal neurons transfected with the indicated constructs as shown in $C .{ }^{* * *} p<0.001$, one-way ANOVA with Bonferroni's multiple-comparisons test. The number labeled in the graph indicates the number of neurons analyzed. Error bars indicate SEM. $\boldsymbol{F}$, Western blot (left) and statistical analysis (right) of rat cortical neurons infected with lentivirus containing nonsilencing shRNA (NS) or CDYL shRNA. The efficiency of CDYL knock-down is about 50\%. G, Left, Comparison of the target sequence between WT-CDYL and shRNA-resistant CDYL (CDYL-R). The third nucleotide in each codon was mutated in CDYL-R without altering the identity of amino acids. Right, Representative Western blotting of FLAG-CDYL in protein lysates from 293T cells transfected with CDYL shRNA plus CDYL-R. $\boldsymbol{H}$, Representative images of hippocampal neurons transfected at DIV 8 for $3 \mathrm{~d}$ with nonsilencing shRNA (NS), CDYL shRNA, CDYL shRNA + PBK vector, CDYL shRNA + CDYL-R, or CDYL shRNA + CDYL del60-Ras indicated. Cells were cotransfected with GFP to visualize the morphology of the transfected neurons. Scale bar, $50 \mu \mathrm{m}$. I, Sholl analysis of hippocampal neurons as shown in $\boldsymbol{H}$. $\boldsymbol{J}$, Quantification of the total dendritic branch length of hippocampal neurons as shown in $\boldsymbol{H}$. The methods used were as in E. In this and all subsequent figures, the numbers of neurons are as indicated in graphs of Sholl analysis and total dendritic branch length. 


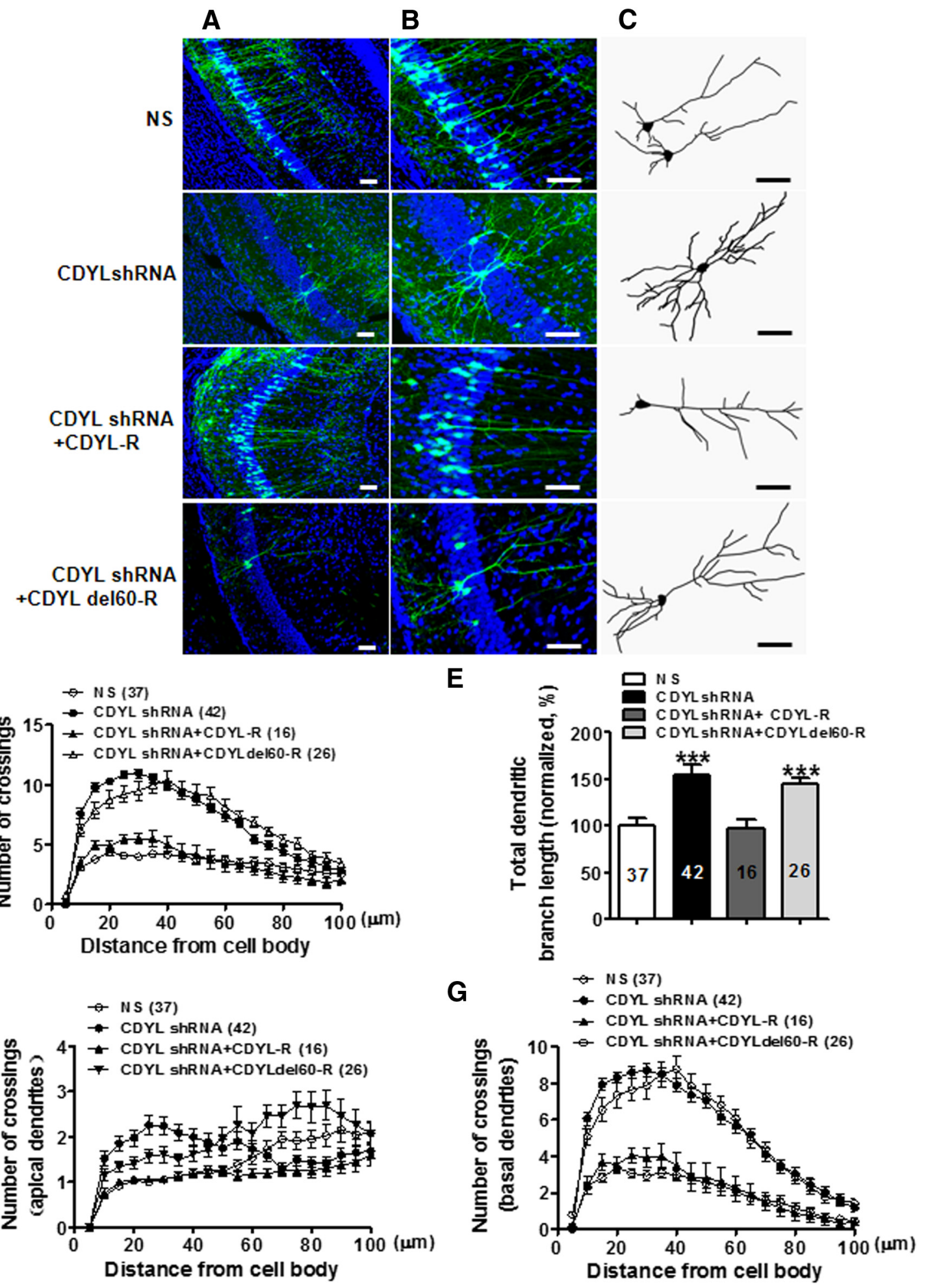

Figure 2. CDYL restricts dendritic morphogenesis in vivo. $A$, Representative images of $\mathrm{P} 15$ mice brains, which were transfected with GFP and the indicated constructs by in utero electroporation at E14.5. Transfected cells were visualized by staining coronal slices with GFP antibody and Hoechst stain. Scale bar, $50 \mu \mathrm{m} . \boldsymbol{B}, \boldsymbol{C}$, Representative higher-magnification images (B) and neurolucida tracing (C) of hippocampal neurons as in $\boldsymbol{A}$. Scale bar, $50 \mu \mathrm{m}$. $\boldsymbol{D}$, Sholl analysis of the neurons from hippocampal slices from in utero electroporated brains transfected with the indicated constructs. Analysis was done at a distance of $100 \mu \mathrm{m}$ from the soma. $\boldsymbol{E}$, Quantification of the total dendritic branch length of the neurons from hippocampal slices. $\boldsymbol{F}, \boldsymbol{G}$, Sholl analysis of apical $(\boldsymbol{F})$ and basal dendrites (G) was performed, respectively.

restored CDYL-inhibited dendrite complexity (Fig. 4A-C), whereas blocking TrkB, the major cell surface receptor of BDNF (Park and Poo, 2013), by recombinant TrkB-Fc in neurons alleviated the increase of dendritic branching caused by CDYL
shRNA (Fig. 4D-F). BDNF is a secreted neurotrophic factor that can exert its function in both a paracrine and an autocrine fashion (Horch and Katz, 2002; Bloodgood et al., 2013; Sylwestrak and Scheiffele, 2013). In our system, alteration of BDNF in a small 
A

defense response

response to organic substance

synaptic transmission

behavior

neuron differentiation

transmission of nerve impulse

inflammatory response

immune response

response to temperature stimulus

cell-cell signaling

regulation of neurological system process

cell motion

di-, tri-valent inorganic cation homeostasis

response to wounding

regulation of transmission of nerve impulse

positive regulation of cell communication

response to prote in stimulus

response to abiotic stimulus

neuron development

response to extracellu lar stimulus

chemical homeostasis

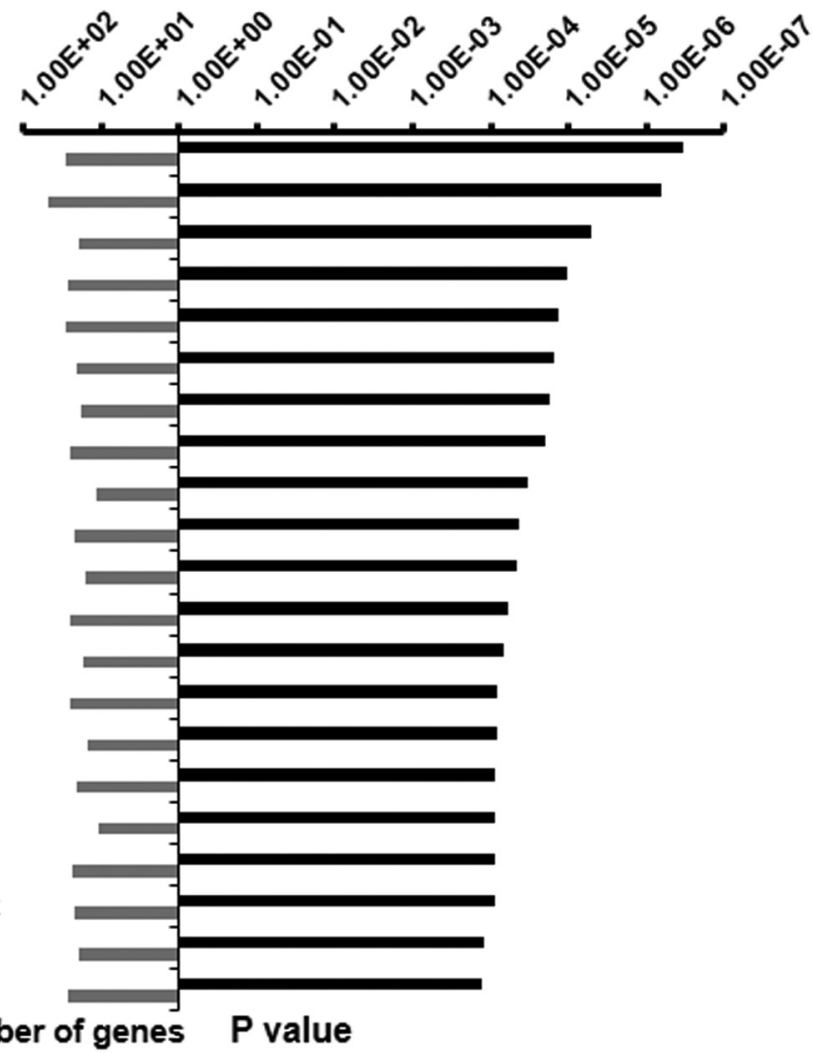

B

\begin{tabular}{ll}
\hline pathway & gene \\
\hline defense response & A2m \\
response to organic substance & Cd83 \\
& Anxa1 \\
& Txnip \\
& H2-M3 \\
& Gipr \\
& Bdnf \\
synaptic transmission & Tac1 \\
Behavior & Fos \\
& Cdkn1c \\
neuron differentiation & Stat3 \\
& Gpc2 \\
transmission of nerve impulse & Egr2 \\
& Bdnf \\
& Tac1 \\
\hline
\end{tabular}
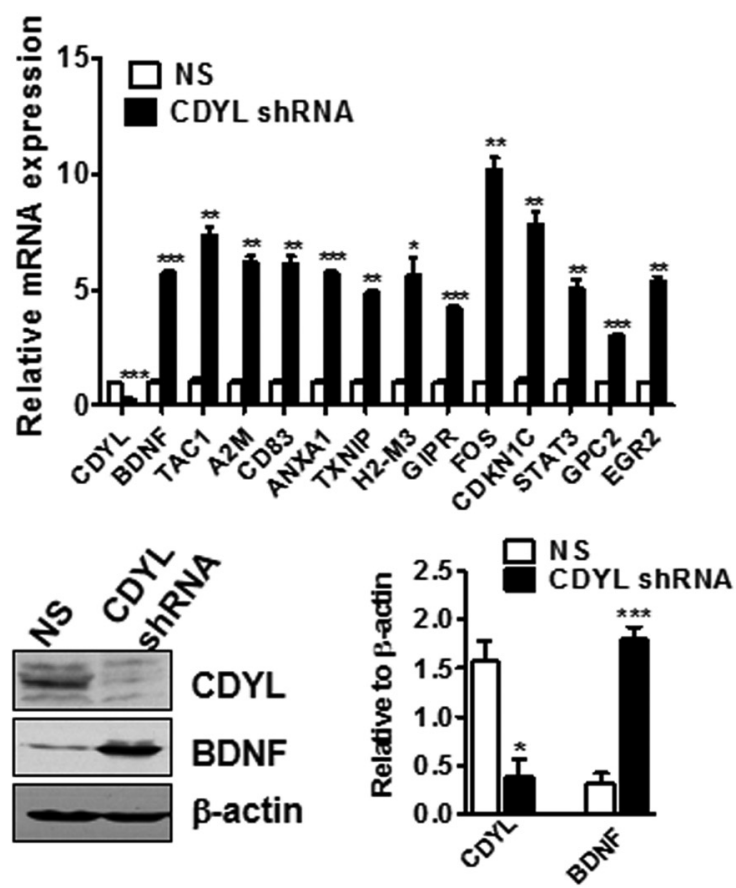

Figure 3. Genome-wide identification of CDYL target genes in cortical neurons. $A$, Genome-wide microarray analysis was performed in cultured primary rat cortical neurons under CDYL depletion via lentiviral infection with CDYL shRNA construct. G0 analysis showed that many genes that were upregulated upon CDYL knock-down are involved in neurological functions. B, Quantitative real-time RT-PCR analysis of the representative genes from each of the six most upregulated functional clusters of the upregulated genes upon CDYL knock-down. Cortical neurons were infected with lentivirus containing CDYL shRNA or nonsilencing shRNA (NS) for $7 \mathrm{~d}$. The protein levels of BDNF and CDYL were examined by Western blot analysis (bottom left) and quantified (bottom right). The experiments were repeated three times and Student's $t$ test was used for statistical analysis. ${ }^{*} p<0.05 ;{ }^{* *} p<0.01 ;{ }^{* * *} p<0.001$.

percentage of cells by overexpression or knock-down of CDYL was sufficient to change the dendrite branching pattern in these cells, suggesting that BDNF acts in a cell-autonomous manner under these circumstances. Indeed, compared with hippocampal neurons transfected with control siRNAs, BDNF siRNAtransfected neurons showed fewer dendritic arbors and less total dendritic branch length (Fig. $5 B-D$ ). In addition, BDNF siRNAs abolished the effects of CDYL shRNA on dendritic complexity, 

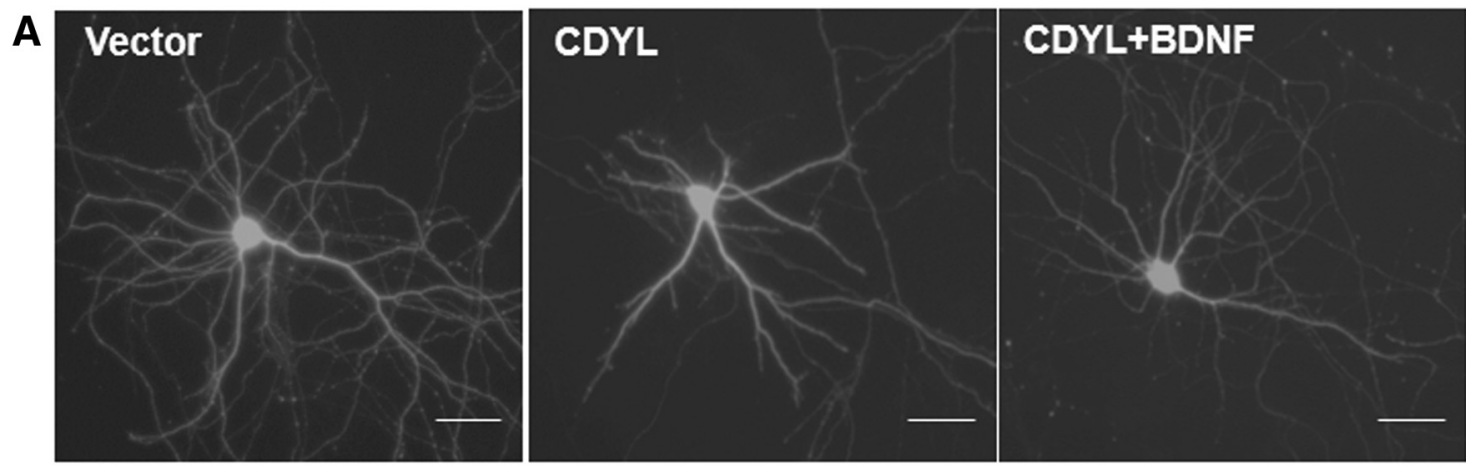

B

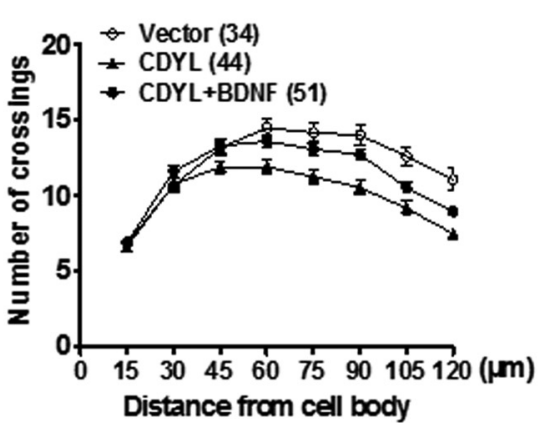

D
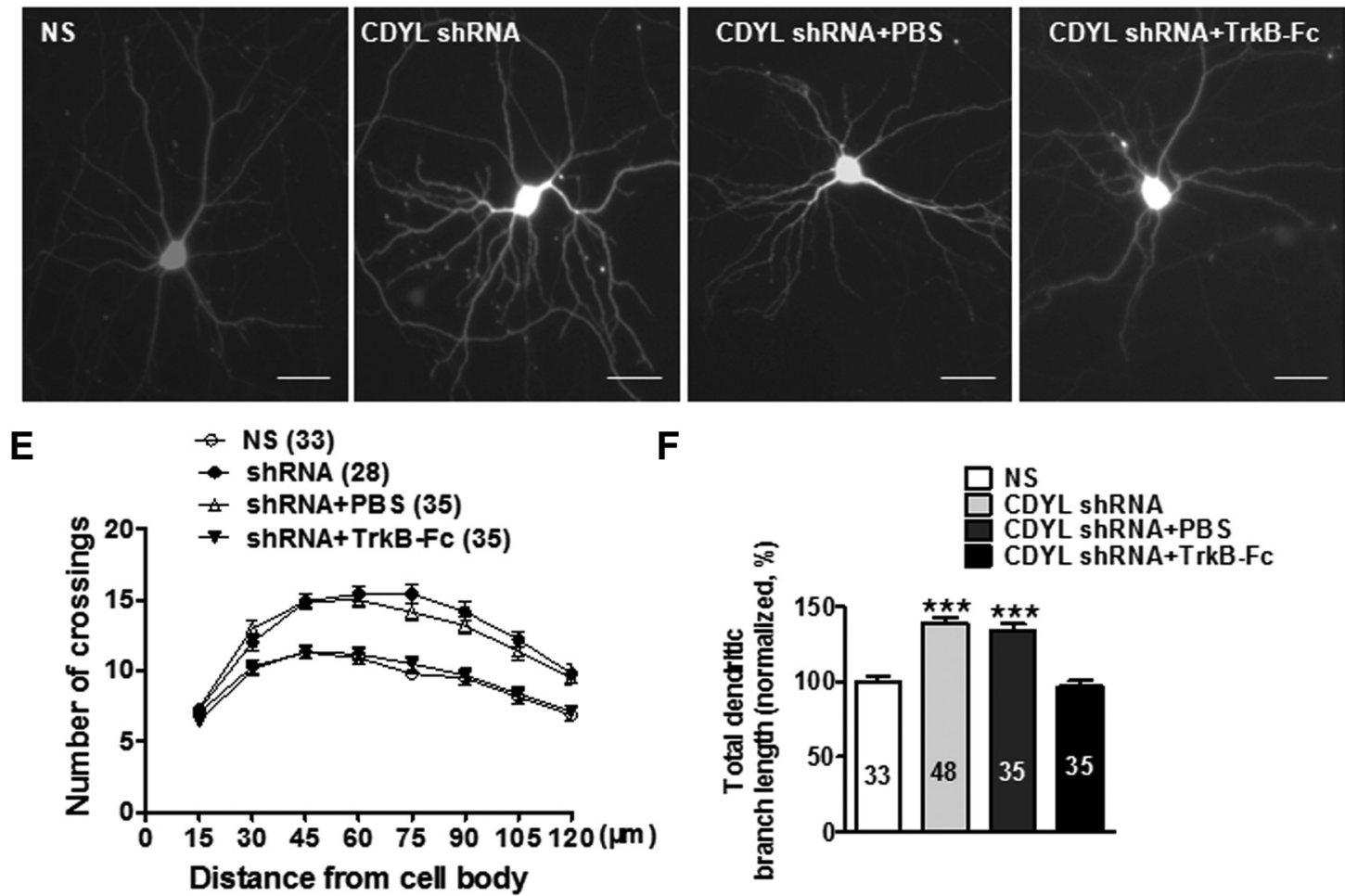

$\mathbf{F}$

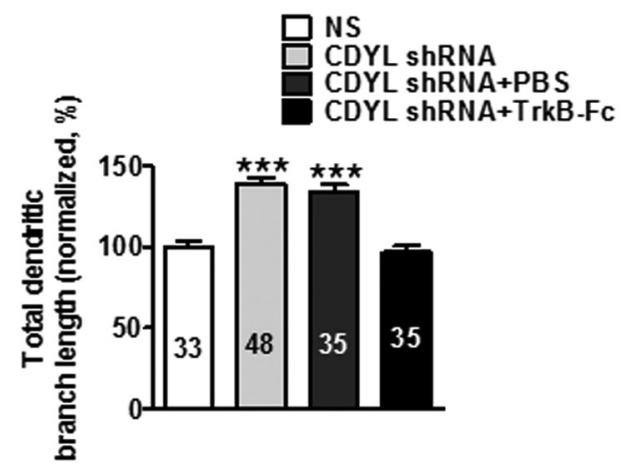

Figure 4. BDNF is required for the effects of CDYL on dendritic branching. $\boldsymbol{A}$, Representative images of hippocampal neurons transfected with the control PBK or the CDYL construct plus GFP at DIV 8 . After $12 \mathrm{~h}$, BDNF ( $25 \mathrm{ng} / \mathrm{ml}$ ) was added to the transfected neurons and the cells continued to grow for $3 \mathrm{~d}$ before the images were collected. Scale bar, $50 \mu \mathrm{m}$. $\boldsymbol{B}, \boldsymbol{C}$, Sholl analysis $(\boldsymbol{B})$ and quantification $(\boldsymbol{C})$ of total dendritic branch length of the neurons as shown in $\boldsymbol{A}$. The methods used in $\boldsymbol{C}$ were as in Figure $1 E$. $\boldsymbol{D}$, Representative images of cultured hippocampal neurons cotransfected with GFP and nonsilencing shRNA (NS) or CDYL shRNA at DIV 8 . After $12 \mathrm{~h}$, transfected neurons were treated with $0.33 \mu \mathrm{g} / \mathrm{ml}$ TrkB-Fc for $3 \mathrm{~d}$ before the images were collected. Scale bar, $50 \mu \mathrm{m}$. $\boldsymbol{E}, \boldsymbol{F}$, Sholl analysis $(\boldsymbol{E})$ and quantification $(\boldsymbol{F})$ of total dendritic branch length of the neurons as shown in $\boldsymbol{D}$. The methods used in $\boldsymbol{F}$ were as in Figure $1 E$.

further demonstrating that autocrined BDNF is required for CDYL-mediated regulation of dendritic branching (Fig. $5 E-G$ ). Together, our data suggest that CDYL restricts dendrite morphogenesis through inhibition of BDNF expression.
CDYL suppresses BDNF transcription by recruiting H3K27 methyltransferase activity

We investigated the molecular basis for CDYL-regulated BDNF transcription to further understand the role of CDYL in dendrite 
A

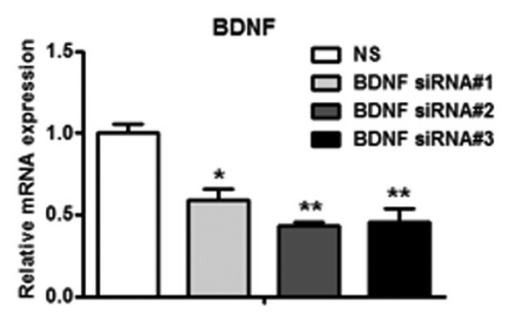

B
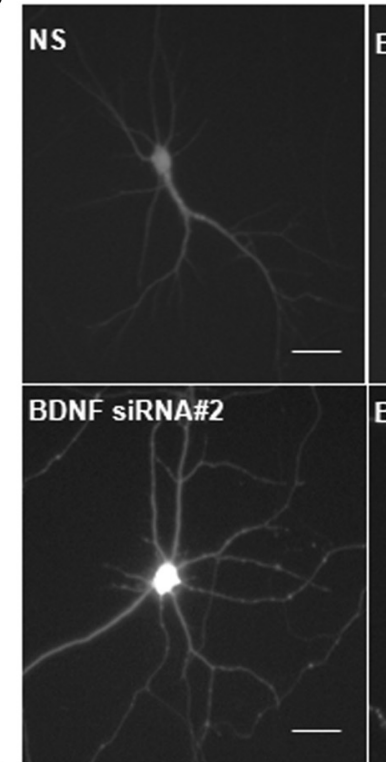

E

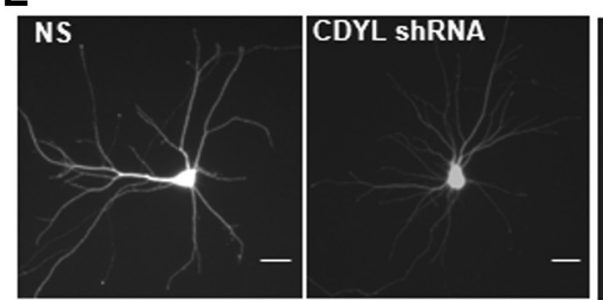

$\mathbf{F}$

\section{BDNF siRNA\#1}

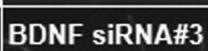

$\rightarrow$ NS (31)

- CDYL shRNA (22)

+ CDYL Sh RNA+BDNF SiRNA\#1 (30)

g) 207 CDYL ShRNA+BDNF SIRNA\#2 (30)

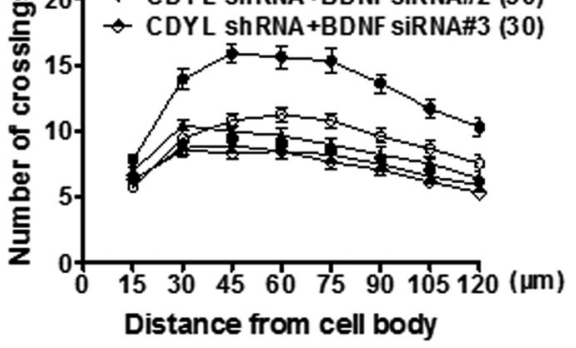

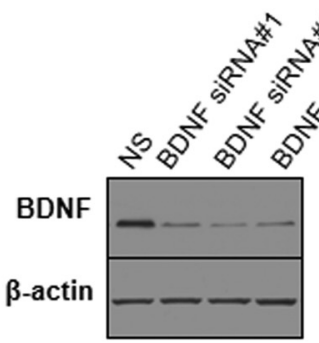

C

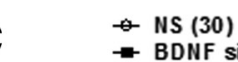

- BDNF SiRNA\#1 (30)

I BDNF SiRNA\#2 (26)

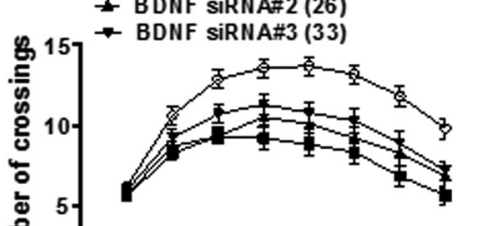

D

Distance from cell body

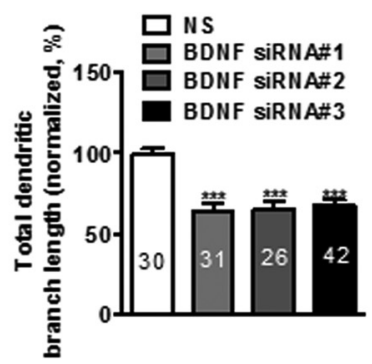

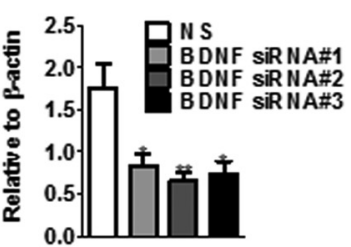

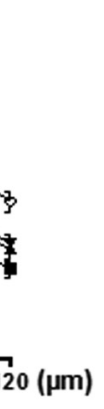$$
\text { (n) }
$$ 
$B D N F$, by its association with epigenetic enzyme(s) catalyzing the addition of repressive histone mark(s). Recently, it was found that epigenetic factor MeCP2, a transcriptional corepressor that recognizes methylated cytosines within DNA, suppresses dendrite morphogenesis and synapse development and behavior through a genome-wide chromosomeremodeling mechanism (Cohen et al., 2011). Therefore, we first isolated chromatin histones by acid extraction from cultured neurons under CDYL depletion via lentiviral infection with CDYL shRNA and analyzed the global levels of H3K9me3, H3K27me3, and H3Ac (acetylation) by Western blotting. No significant changes were found in the global levels of these modifications in CDYLdepleted neurons (Fig. 6A), suggesting that CDYL does not likely affect the overall chromatin modification pattern and may instead act in a gene-specific manner. In our previous ChIP-Seq study with CDYL-specific polyclonal antibodies (Zhang et al., 2011), we found a significant enrichment of CDYL-binding signals in the promoter region (promoter II) of $B D N F$, which is conserved in rat and human (Mortazavi et al., 2006; Tahiliani et al., 2007; Zhang et al., 2011; Fig. 6B). Remarkably, the neuron-restrictive silencer element (NRSE, also known as RE1), the binding sequence for the transcriptional repressor REST, is also present in this region. Therefore, we hypothesized that CDYL recruits repressive histone modification enzymes to this regulatory element to repress $B D N F$ transcription.

To investigate which histone modification activity associated with CDYL in this region is most important for $B D N F$ transcription, we performed ChIP assays with soluble chromatin extracted from SH-SY5Y cells using specific antibodies against CDYL, H3K9me3, H3K9me2, H3K27me3, pan-acetylation, and REST. SY5Y cells were used in this assay because none of the commercially available CDYL antibodies, including a polyclonal antibody that we previously generated, were suitable to perform ChIP experiments in rat neurons. We found that, upon knock-down of CDYL, the level of H3K27me3, but not that of histone acetylation, $\mathrm{H} 3 \mathrm{~K} 9 \mathrm{me} 3$ or $\mathrm{H} 3 \mathrm{~K} 9 \mathrm{me} 2$, was decreased dramatically (Fig. 6C). The recruitment of the sequence-specific transcription repressor REST to this site was not affected by knock-down of CDYL. We validated CDYLinhibited BDNF transcription in SY5Y cells by real-time RT-PCR analysis (Fig. 6D). It is known that the transcription of $B D N F$ is driven by multiple promoters (Liu et al., 2006; Pruunsild et al., 2007). Knock-down of CDYL most significantly resulted in derepression of the promoter II and the upregulation of BDNF2B and BDNF2C transcripts, which start from exon IIA and IIB, respectively. Together, these results support an argument that CDYL inhibits $B D N F$ transcription mainly through

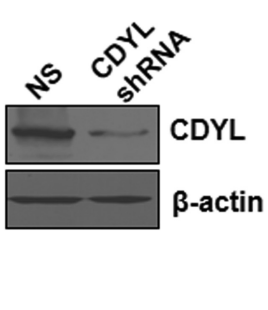

CDYL Binding Sites

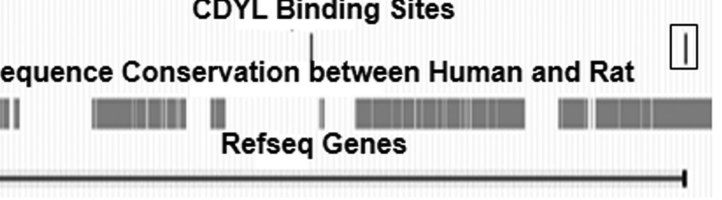

D

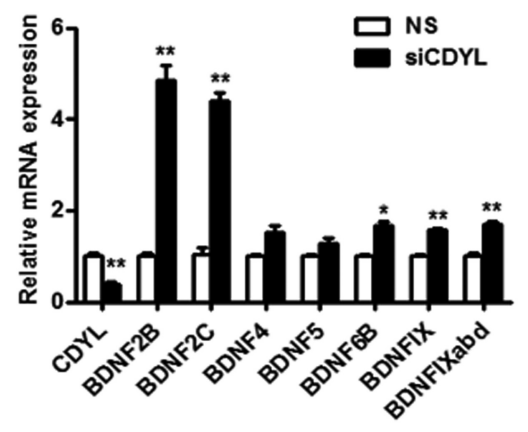

Figure 6. CDYL suppresses BDNF transcription by recruiting H3K27 methyltransferase activity. $A$, Cortical neurons were infected the dense mode in the middle track. C, SH-SY5Y cells were transfected with control siRNA or CDYL-specific siRNA. Seventy-two 作 levels of BDNF transcripts and CDYL were measured by quantitative real-time RT-PCR. The experiments were repeated for at least three times and Student's $t$ test was used for statistical analysis.

recruiting the $\mathrm{H} 3 \mathrm{~K} 27$ methyltransferase activity to the promoter of the gene.

\section{Coordinated regulation of dendrite complexity by CDYL and $\mathrm{EZH} 2$}

We have demonstrated previously that CDYL interacts directly with EZH2, the catalytic subunit of the PRC2 complex, and may facilitate the establishment and propagation of repressive H3K27me3 mark along chromatin (Zhang et al., 2011). CDYL inhibits BDNF transcription mainly through recruiting the H3K27 methyltransferase activity to the gene promoter, suggesting that EZH2 likely plays an important role in CDYL-inhibited BDNF transcription and thus the effect of CDYL on dendritic branching. We first confirmed the interaction between CDYL and EZH2 in neurons. FLAG-tagged CDYL protein, which was purified from baculovirus-infected sf9 cells and coupled to an anti-FLAG M2 affinity gel, was incubated with rat brain lysates and EZH2 signal was clearly 
A

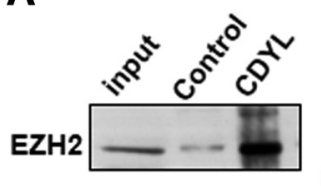

B

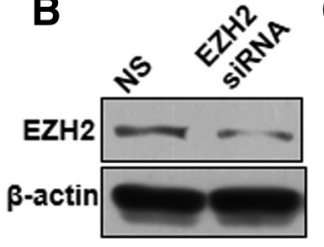

C

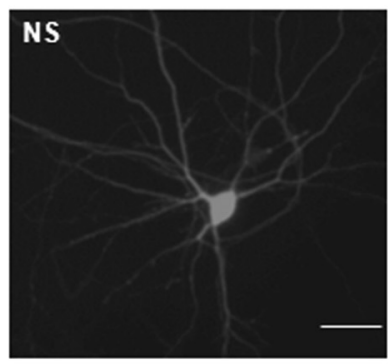

$\mathbf{F}$ h-BDNF promoter

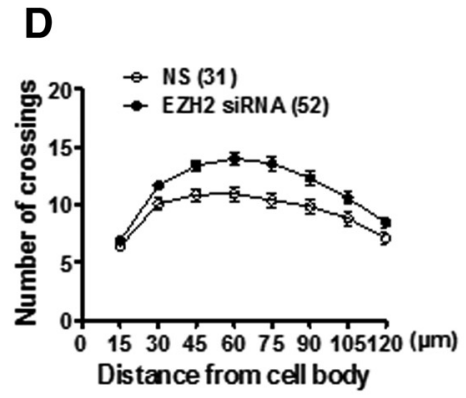

E

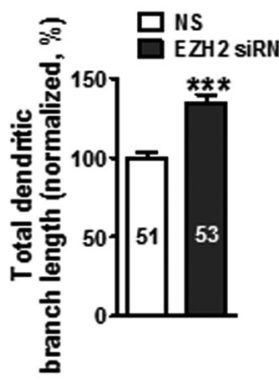

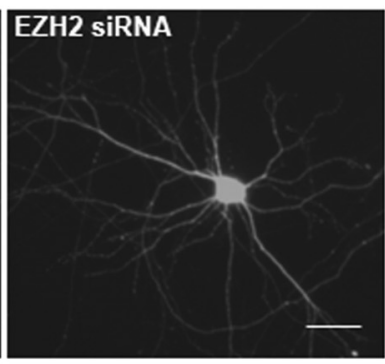

G

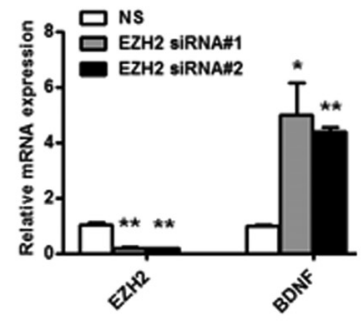

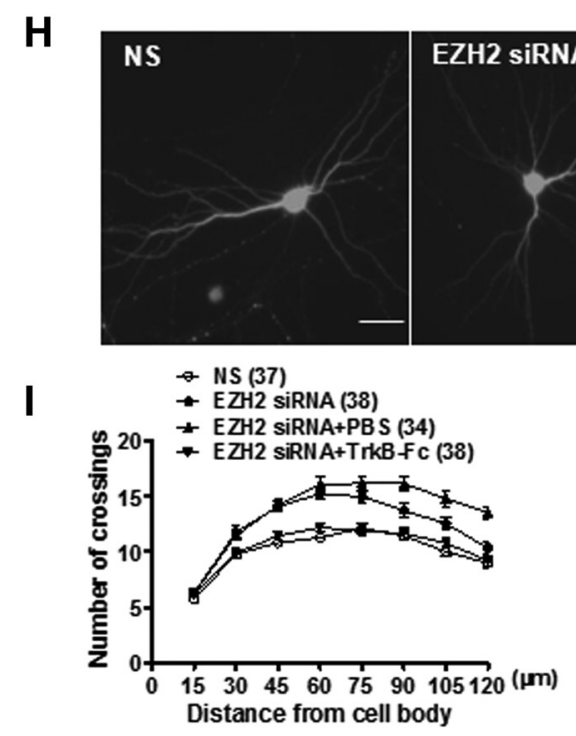
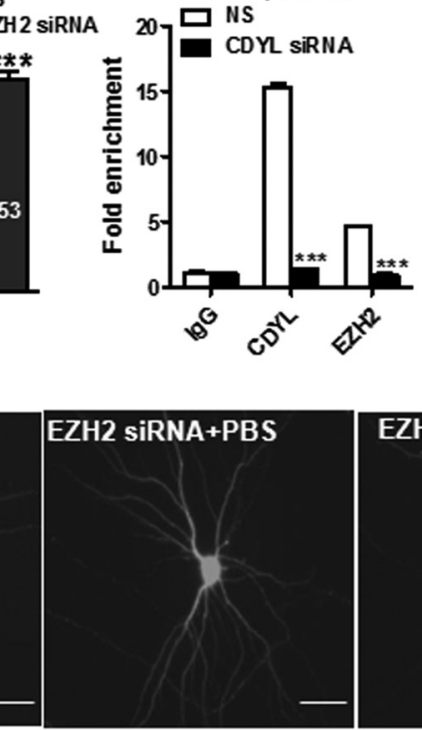

EZH2 siRNA+TrkB-Fc

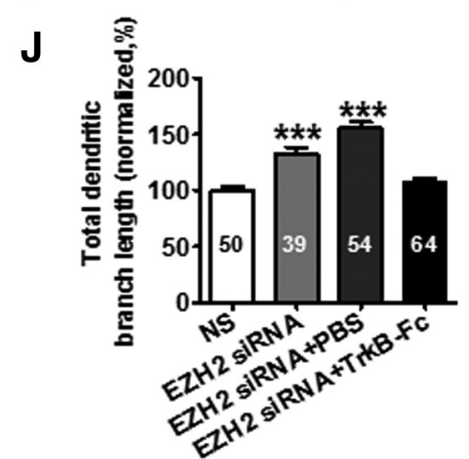

Figure 7. EZH2 participates in the regulation of dendritic complexity. $A, C D Y L$ interacts with EZH2 in the brain. FLAG-CDYL protein was purified from sf9 cells with baculovirus expression system. Purified proteins were immobilized on anti-FLAG M2 affinity gel (Sigma-Aldrich) and incubated with rat brain lysates. Bound proteins were detected with anti-EZH2 antibodies. B, EZH2 expression was efficiently knocked down by specific siRNAs. Nonsilencing or EZH2-siRNAs were transfected into rat $\mathrm{C} 6$ cells. Total cell lysates were collected after $48 \mathrm{~h}$ and the protein levels of EZH2 were examined by Western blot analysis. C, Representative images of neurons transfected with the indicated constructs at DIV 8 for $3 \mathrm{~d}$. Scale bar, $50 \mu \mathrm{m}$. D, Sholl analysis of neurons as shown in $\boldsymbol{C}$. $\boldsymbol{E}$, Quantification of total dendritic branch length of the neurons as shown in $C .{ }^{* * *} p<0.001$, Student's unpaired two-tailed t test. Error bars indicate SEM. F, SH-SY5Y cells were transfected with control siRNA or CDYL specific siRNA. Seventy-two hours after the transfection, cell lysates were collected and ChIP experiments were performed using the indicated antibodies. Real-time PCR assays were performed for the measurement. The experiments were repeated three times and Student's $t$ test was used for statistical analysis. G, EZH2 siRNA (50 nM) was transfected into SH-SY5Y cells. Total RNA was extracted $48 \mathrm{~h}$ later and the total mRNA levels of BDNF and CDYL were measured by quantitative real-time RT-PCR. The experiments were repeated at least three times and Student's $t$ test was used for statistical analysis. $\boldsymbol{H}$, Representative images of cultured hippocampal neurons cotransfected with GFP and nonsilencing shRNA (NS) or EZH2 siRNA. The cells were treated with PBS or TrkB-Fc as in Figure $4 D$. Scale bar, $50 \mu \mathrm{m}$. $\boldsymbol{I}, \boldsymbol{J}$, Sholl analysis $(\boldsymbol{I})$ and quantification $(\boldsymbol{J})$ of total dendritic branch length of the neurons as shown in $\mathbf{G} .{ }^{* * *} p<0.001$, one-way ANOVA with Bonferroni's multiple-comparisons test.

detected in CDYL-precipitated protein complexes (Fig. 7A). Similar to knock-down of CDYL, knock-down of EZH2 in cultured hippocampal neurons led to a dramatic increase in the dendritic complexity of these cells, supporting the physical interaction between CDYL and EZH2 and a functional connection between these two epigenetic factors (Fig. $7 C-E$ ). Knock-down of CDYL dramatically decreased the association between EZH2 and the promoter of BDNF (Fig. $7 F$ ) and knock-down of EZH2 with specific siRNAs significantly increased the expression of BDNF (Fig. 7G), further demonstrating that CDYL recruits EZH2 to the BDNF promoter to inhibit gene transcription. TrkB-Fc treatment effectively alleviated the increased dendritic complexity in EZH2-knockdown neurons (Fig. $7 \mathrm{H}-\mathrm{J}$ ), suggesting that EZH2 restricted 
A

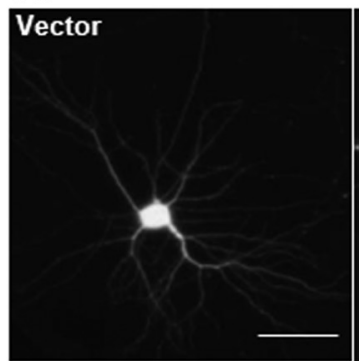

D

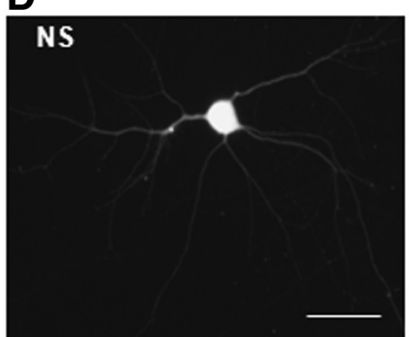

CDYL shRNA+Vector

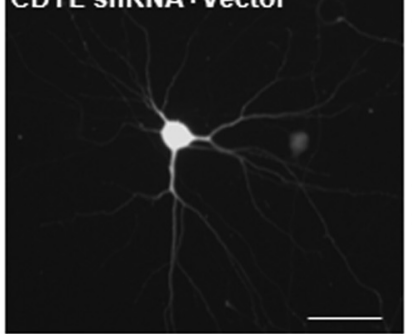

G

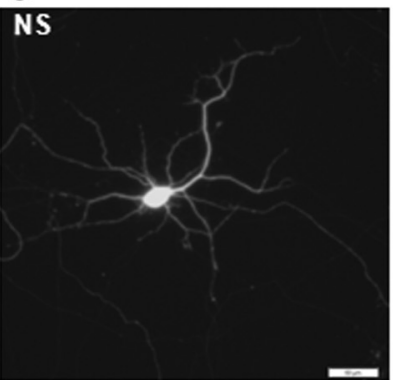

EZH2 siRNA+Vector

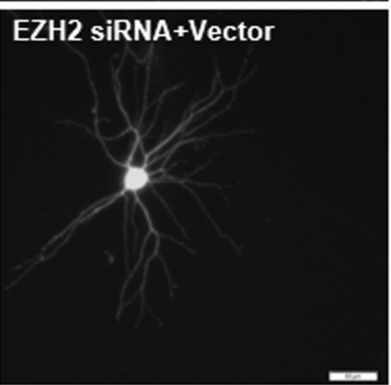

B

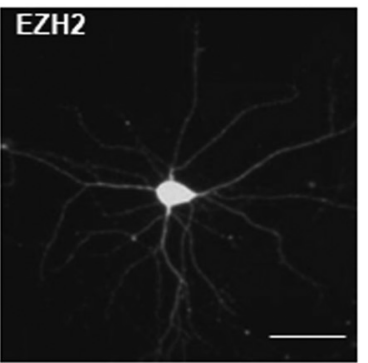

CDYL ShRNA

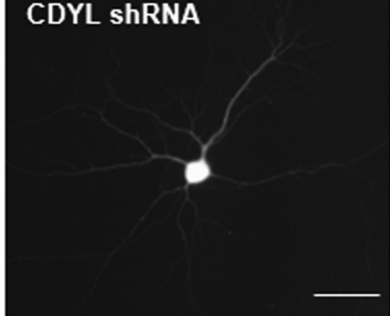

CDYL shRNA+EZH2
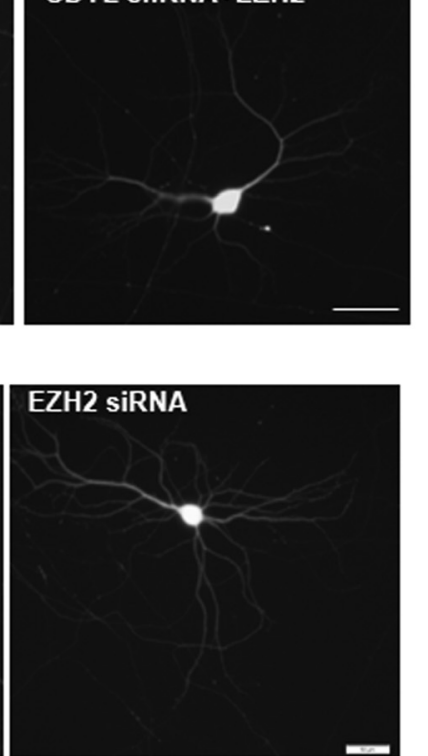

$E Z H 2$ siRNA+CDYL

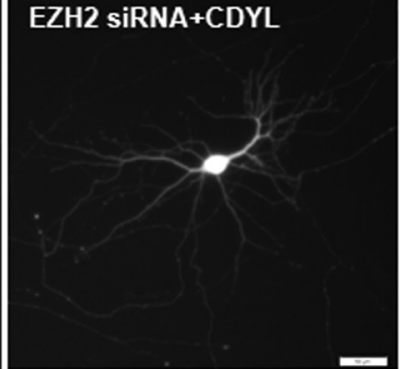

+ Vector (44)

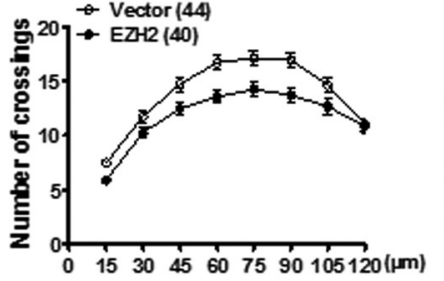

Distance fromcell body

\section{E}

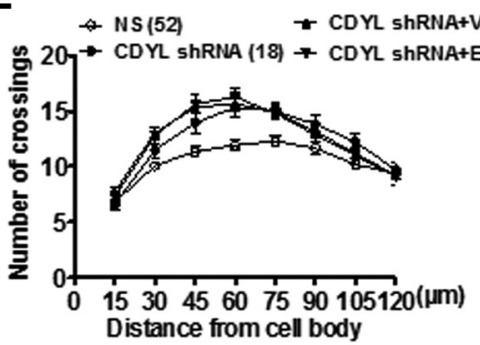

$\mathbf{F}$

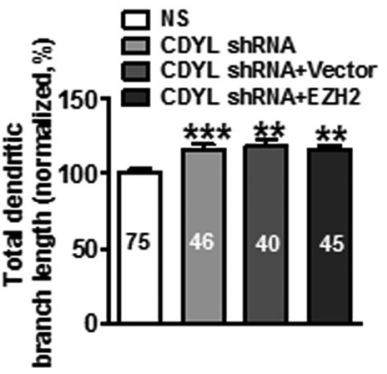

H

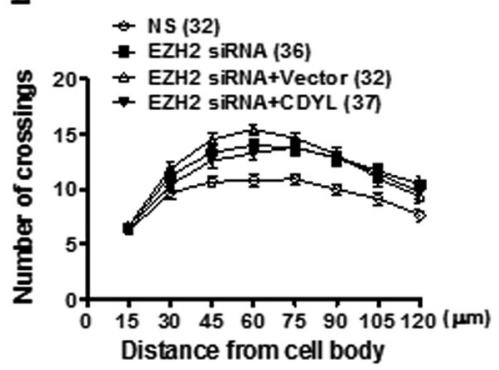

|

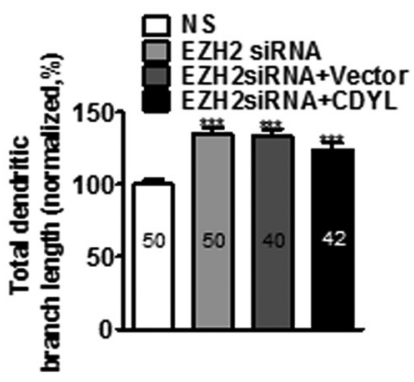

Figure 8. CDYL and EZH2 coordinately regulate dendritic complexity in an interdependent manner. $\boldsymbol{A}$, Representative images of neurons transfected with the empty vector or the EZH 2 construct at DIV 8 for 3 d. Scale bar, $50 \mu \mathrm{m}$. B, C, Sholl analysis $(\boldsymbol{B})$ and quantification $(\boldsymbol{C})$ of total dendritic branch length of the neurons as shown in $\boldsymbol{A}$. ${ }^{*} p<0.05$, Student's unpaired two-tailed $t$ test. Error bars indicate SEM. $\boldsymbol{D}$, Representative images of neurons transfected with nonsilencing shRNA (NS), CDYL shRNA, CDYL shRNA + vector, or CDYL shRNA + EZH2 at DIV8 for 3 d. Scale bar, $50 \mu \mathrm{m}$. $\boldsymbol{E}, \boldsymbol{F}$, Sholl analysis $(\boldsymbol{E})$ and quantification $(\boldsymbol{F})$ of total dendritic branch length of the neurons as shown in $\boldsymbol{D} .{ }^{* *} p<0.01,{ }^{* * *} p<0.001$, one-way ANOVA with Bonferroni's multiple-comparisons test. Error bars indicate SEM. G, Representative images of neurons transfected with NS, EZH2 siRNA, EZH2 siRNA + vector, or EZH2 siRNA + CDYL at DIV 8 for 3 d. Scale bar, $50 \mu \mathrm{m}$. $\boldsymbol{H}, \boldsymbol{I}$, Sholl analysis $(\boldsymbol{H})$ and quantification $(I)$ of total dendritic branch length of the neurons as shown in $G .{ }^{* * *} p<0.001$, one-way ANOVA with Bonferroni's multiple-comparisons test. Error bars indicate SEM.

dendritic branching through the BDNF pathway. Moreover, although overexpression of EZH2 decreased dendritic branching of hippocampal neurons to the same extent as overexpression of CDYL (Fig. $8 A-C$ ), overexpression of EZH2 in
CDYL-depleted hippocampal neurons did not rescue the increased dendritic complexity (Fig. $8 D-F$ ). Conversely, overexpression of CDYL had no effect on the dendritic branching pattern in EZH2-depleted hippocampal neurons (Fig. 8G-I). 
Clearly, CDYL and EZH2 are interdependent in the regulation of dendrite morphology. If our interpretation is correct, this means that CDYL and EZH2 are functionally coordinated in controlling dendrite patterning.

\section{Neural activity increases dendritic complexity through CDYL degradation}

Appropriate dendrite patterning is critical for information processing. Indeed, dendrite arborization increases upon neural activation (Wong and Ghosh, 2002) in response to developmental and environmental cues. Here, the expression level of CDYL gradually decreased as the nervous system matured (Fig. 1B), indicating that the level of CDYL might be regulated by neural activity during development. To understand the dynamics of CDYL in response to external stimulation, cultured neurons were treated with $\mathrm{KCl}$, which causes an increase of cellular calcium influx (Sin et al., 2002; Peng et al., 2009; Wills et al., 2012). Although $\mathrm{KCl}$ stimulation of cortical neurons did not change the mRNA level of either CDYL or EZH2 (Fig. 9A), a rapid decrease of the protein level of CDYL, but not that of EZH2, was found in these stimulated cells (Fig. 9B). We therefore examined whether the decrease of CDYL protein is a function of the ubiquitinproteasome protein degradation machinery. Indeed, treatment of cells with MG132 or clasto-lactacystin $\beta$-lactone, two widely used proteasome inhibitors, abrogated the decrease in CDYL protein levels in these $\mathrm{KCl}$-stimulated neurons (Fig. 9D, E), suggesting that proteasome-mediated protein degradation is responsible for the rapid depletion of CDYL. Presumably, the rapid clearance of CDYL, which happens within 30 minutes after $\mathrm{KCl}$ stimulation, unleashes the inhibitory effect on BDNF expression and is responsible, at least in part, for the upregulation of BDNF mRNA (Fig. 9A) and the increased dendritic complexity in $\mathrm{KCl}$ stimulated neurons. Indeed, $\mathrm{KCl}$ stimulation of cultured neurons led to increased dendritic complexity concomitant with the decrease of CDYL and increase of BDNF (Fig. 9F-J). TrkB-Fc treatment efficiently blocked $\mathrm{KCl}$-induced dendritic branching (Fig. $9 F-H)$, suggesting that increased BDNF expression, which is at least partially caused by CDYL degradation, mediates neuralactivity-induced dendritic complexity.

\section{Discussion}

In the present study, we identified the transcriptional repressor CDYL as a novel regulator of dendrite morphogenesis. In cultured primary hippocampal neurons, overexpression of CDYL reduces the dendritic arborization and total dendritic branch length; conversely, knock-down of CDYL increases the dendritic complexity, which could be counteracted by overexpression of a shRNA-resistant CDYL construct. In utero electroporation of CDYL shRNA leads to an increased dendritic complexity in vivo. DNA microarray analysis revealed that CDYL represses a number of genes that constitute several important cellular pathways pertinent to neurological functions, including synaptic transmission, response to various stimuli, and neuron differentiation. Among the target genes of CDYL, the neurotrophin BDNF is a key player in promoting dendrite development. By inhibiting the transcription of BDNF, CDYL acts as an internal barrier to suppress inappropriate and untimely dendrite branching. During brain maturation, the CDYL protein level decreases. CDYL is rapidly degraded by proteasome upon increase of calcium influx in neurons, thereby unleashing the expression of BDNF to adapt dendrite growth in these circumstances.

The region surrounding NRSE at the promoter II of BDNF has been demonstrated to play a key role in the regulation of the transcription of BDNF (Timmusk et al., 1999; Zuccato et al., 2003). It is reported that this region is responsive to wild-type huntingtin, which inhibits the silencing activity of NRSE through cytoplasmic sequestration of repressor REST, an important control mechanism lost in the pathology of Huntington disease (Zuccato et al., 2003). Based on our observation that CDYL is rapidly degraded upon $\mathrm{KCl}$ addition, it is likely that the NRSE at the promoter II of $B D N F$ could also play an important role in response to acute environmental signals including $\mathrm{KCl}$ stimulation. In view of a previous report that acute cocaine administration specifically increases the expression of a BDNF4 splice variant (Liu et al., 2006), it becomes increasingly possible that different environmental signals entail the transcription of distinct BDNF transcripts.

In our previous ChIP-Seq analysis performed in nonneuronal cells, we found significant enrichment of CDYLbinding signals surrounding NRSE, the consensus REST-binding sequences (Zhang et al., 2011). GO analysis indicated that a significant number of CDYL target genes are involved in the development and functionality of the nervous system. Protein-protein interaction between CDYL and REST was reported by other groups (Mulligan et al., 2008) and REST is considered to be the major transcriptional repressor acting to silence neuronal genes in non-neuronal cells (Chen et al., 1998; Mortazavi et al., 2006). However, analysis of Rest-deficient mice indicated that whereas REST is required for the correct development of the nervous system, the expression of neuronal genes in non-neuronal tissues is also lacking, suggesting that alternative mechanisms exist to inhibit ectopic expression of neuron-specific genes (Chen et al., 1998). Given a recent report that iPS cells derived from $C d y l^{-/-}$ mouse fibroblasts are much more prone to spontaneous differentiation to neurons than the cells derived from $\mathrm{Cdyl}^{+/+}$mice (Wan et al., 2013), it is intriguing to speculate that CDYL is an important corepressor of REST to switch off neuronal genes in non-neuronal tissues. Further studies with $C d y l$ knock-out mice and $C d y l / R e s t$ double knock-out mice will be critical to address this issue.

By recognizing H3K27me3 and H3K9me2/3, CDYL functions as a reader protein for histone modifications and participates in the establishment of the repressive histone methylation marks in chromatin (Mulligan et al., 2008; Zhang et al., 2011). In addition, CDYL has been reported to recruit histone deacetylase activity to inhibit target gene expression (Caron et al., 2003). In repression of the transcription of BDNF, change of CDYL level mainly causes alteration of $\mathrm{H} 3 \mathrm{~K} 27 \mathrm{me} 3$ at the BDNF promoter. Moreover, we have demonstrated previously that $\mathrm{H} 3 \mathrm{~K} 9 \mathrm{me} 3$ and H3K27me3 at CDYL target sites are not always concurrent (Zhang et al., 2011), suggesting that CDYL might coordinate with different histone modification enzymes at different genomic loci. CDYL interacts directly with EZH2, the catalytic subunit of the PRC2 complex responsible for the histone H3K27 trimethylation. It has been found PRC2 subunits play an important role in dendrite branching in Drosophila (Parrish et al., 2006; Parrish et al., 2007). We have demonstrated here, for the first time, that EZH2 is a key negative regulator of dendrite morphogenesis in mammalian neurons and that CDYL and EZH2 coordinately inhibit the transcription of $B D N F$ and restrict dendrite arborization. When neuronal cells receive acute environmental signals such as $\mathrm{KCl}$ stimulation, only CDYL, not EZH2, is rapidly degraded to unleash the expression of neurogenic genes such as $B D N F$, suggesting that the regulatory effect of CDYL is dominant over EZH2 under this circumstance. 
A

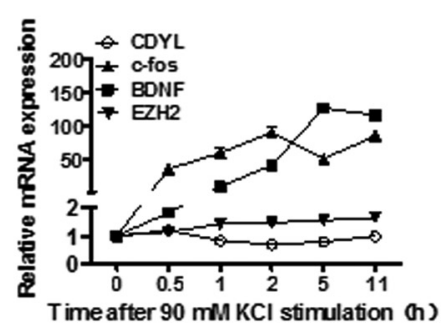

B

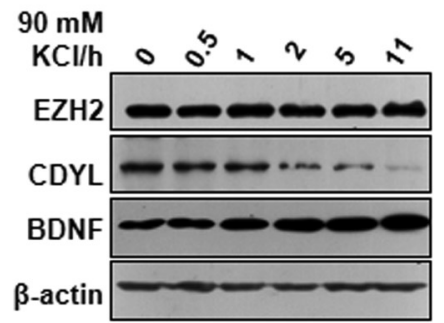

C

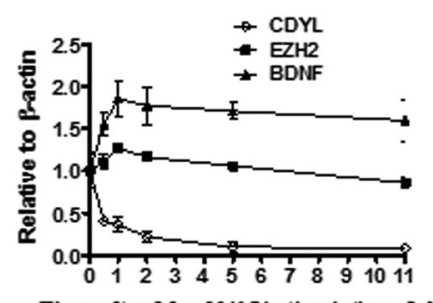

Time after $90 \mathrm{mM} \mathrm{KCl}$ stimulation (h)
D

MG132

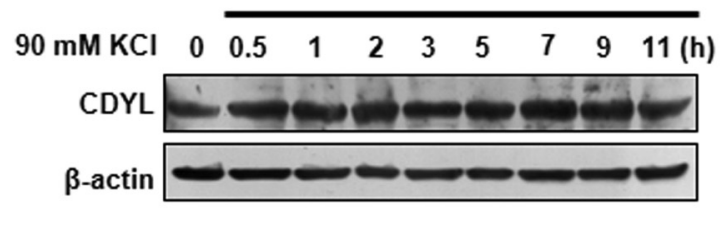

E

\section{Clasto-lactacystin $\beta$-lactone}

$90 \mathrm{mM} \mathrm{KCl}$

CDYL

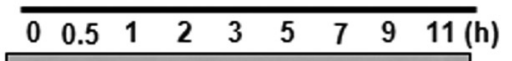

$\beta$-actin

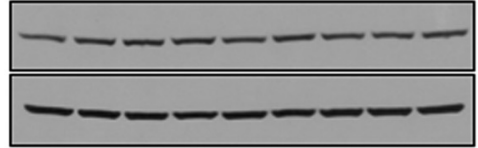

F

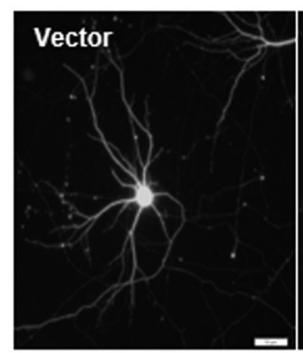

G

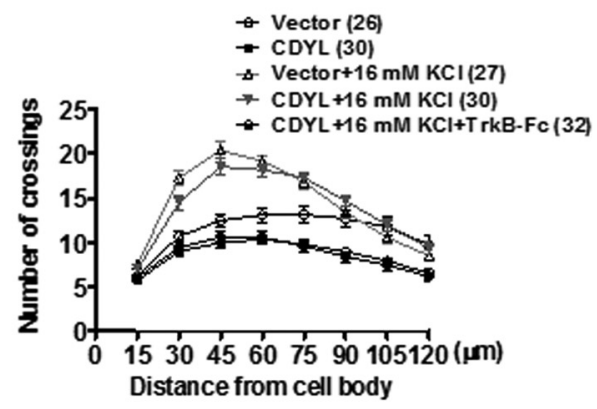

I

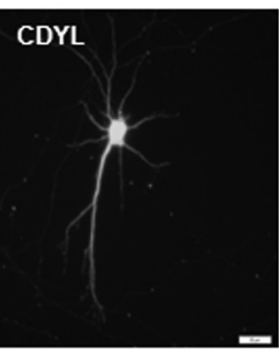

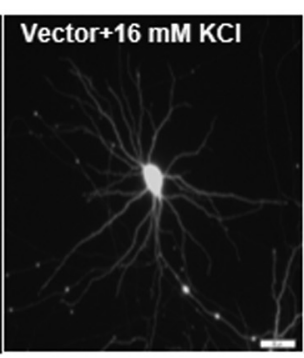
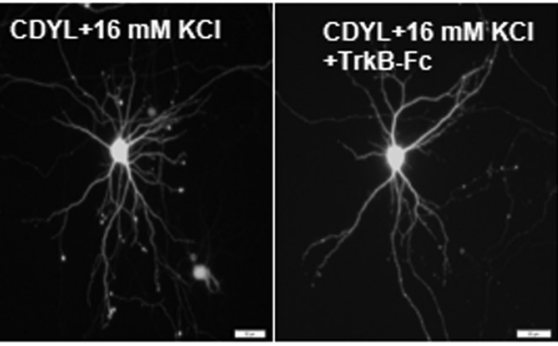

H

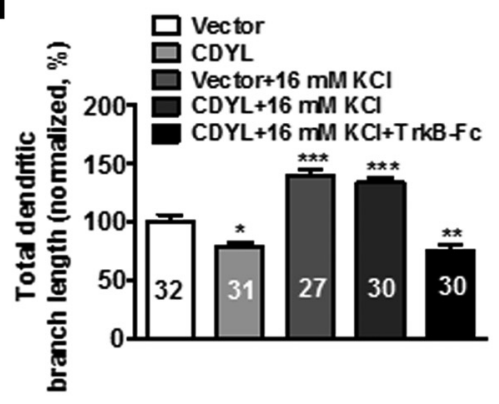

J

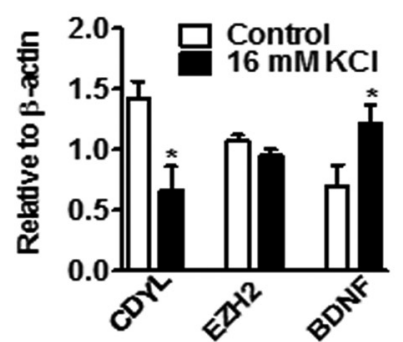

Figure 9. CDYL is rapidly degraded in response to KCl stimulation. $\boldsymbol{A}$, Cortical neurons were treated with $90 \mathrm{~mm} \mathrm{KCl}$ for the indicated times and the $\mathrm{mRNA}$ levels of $\mathrm{c}-\mathrm{fos}$, $\mathrm{CDYL}$, EZH2, and $\mathrm{BDNF}$ were examined by real-time RT-PCR analysis. Expression levels were normalized against that of GAPDH. Each bar represents the mean \pm SEM for triplicate measurements. $\boldsymbol{B}$, Protein lysates were prepared from cultured cortical neurons treated with $90 \mathrm{~mm} \mathrm{KCl}$, and subjected to immunoblot analysis with specific antibodies against CDYL, EZH2, BDNF, and $\beta$-actin. C, Quantification of the protein levels as shown in $\boldsymbol{B}$, which was repeated at least three times. Error bars indicate SEM. $\boldsymbol{D}$, Cortical neurons were treated with $5 \mu \mathrm{m}$ MG132 for $6 \mathrm{~h}$ before stimulating with $90 \mathrm{~mm}$ KCl for the indicated time and protein lysates were extracted and immunoblotted with specific antibodies against CDYL and $\beta$-actin. $\boldsymbol{E}$, Cortical neurons were treated with $20 \mu \mathrm{m}$ clasto-lactacystin $\beta$-lactone overnight before stimulating with $90 \mathrm{~mm} \mathrm{KCl}$ for the indicated times and protein lysates were extracted and immunoblotted with CDYL and $\beta$-actin. $F$, Cultured hippocampal neurons transfected with control or CDYL constructs were treated with or without $16 \mathrm{~mm} \mathrm{KCl}$ in the presence or absence of TrkB-Fc for $2 \mathrm{~d}$ before the morphology was analyzed. Scale bar, $50 \mu \mathrm{m}$. $\boldsymbol{G}, \boldsymbol{H}$, Sholl analysis ( $\boldsymbol{G}$ ) and quantification $(\boldsymbol{H})$ of the total dendritic branch length of the neurons as shown in $\boldsymbol{F} .{ }^{*} p<0.05,{ }^{* *} p<0.01,{ }^{* * *} p<0.001$, one-way ANOVA with Bonferroni's multiple-comparisons test. Error bars indicate SEM. I, J, Cortical neurons were treated with $16 \mathrm{~mm} \mathrm{KCl}$ for $2 \mathrm{~d}$ and the protein levels of CDYL, EZH2, and BDNF were examined by Western blot and quantified. ${ }^{*} p<0.05$, Student's unpaired two-tailed $t$ test. 
Dendrite development is critical to many physiological and pathological conditions in the CNS. Overgrowth of dendrites could lead to abnormal circuit connection and information processing and eventually cause neurological diseases (Mei and Xiong, 2008; Jan and Jan, 2010; Jakovcevski and Akbarian, 2012; Park and Poo, 2013). Conversely, stimulation-induced neuroplasticity, partly due to newly developed dendrite branching, leads to new connections between neurons and is the basis for learning and memory. We have demonstrated that CDYL is an important regulator of dendrite morphogenesis through transcriptional repression. The level of CDYL decreases as the neuron matures and receives $\mathrm{KCl}$ stimulation, suggesting that there might be a neuronal-activity-dependent degradation of CDYL. Whereas it has long been known that neuronal activity leads to upregulation of BDNF and increased dendritic branching, the underlying molecular mechanism remains unclear (Wong and Ghosh, 2002). We have provided evidence that the rapid degradation of CDYL protein during $\mathrm{KCl}$ stimulation could contribute, at least in part, to the increased expression of BDNF by unleashing the inhibitory effect on BDNF transcription. It will be interesting to identify the E3 ligase of CDYL and the corresponding lysine residues of ubiquitin ligation on CDYL. By examining whether overexpression of a CDYL mutant resistant to proteasomal degradation could alleviate $\mathrm{KCl}$-induced dendritic branching, we can further clarify whether CDYL degradation is the major underlying mechanism of the activitydependent BDNF upregulation and the subsequent increase of dendritic branching. It will be also important to clarify the role of CDYL in neural development and neurological diseases using in vivo models such as knock-out mice. Nevertheless, our present findings provide novel insights into the role of CDYL and EZH2 in the regulation of neural development and underpin the importance of epigenetic regulation in the development and functionality of the nervous system. Perhaps more importantly, with the reversible nature of epigenetic modifications, these mechanistic studies may offer better therapeutic opportunities for neurological diseases in the future.

\section{References}

Bloodgood BL, Sharma N, Browne HA, Trepman AZ, Greenberg ME (2013) The activity-dependent transcription factor NPAS4 regulates domainspecific inhibition. Nature 503:121-125. CrossRef Medline

Borrelli E, Nestler EJ, Allis CD, Sassone-Corsi P (2008) Decoding the epigenetic language of neuronal plasticity. Neuron 60:961-974. CrossRef Medline

Caron C, Pivot-Pajot C, van Grunsven LA, Col E, Lestrat C, Rousseaux S, Khochbin S (2003) Cdyl: a new transcriptional co-repressor. EMBO Rep 4:877-882. CrossRef Medline

Chen ZF, Paquette AJ, Anderson DJ (1998) NRSF/REST is required in vivo for repression of multiple neuronal target genes during embryogenesis. Nat Genet 20:136-142. CrossRef Medline

Cohen S, Gabel HW, Hemberg M, Hutchinson AN, Sadacca LA, Ebert DH, Harmin DA, Greenberg RS, Verdine VK, Zhou Z, Wetsel WC, West AE, Greenberg ME (2011) Genome-wide activity-dependent MeCP2 phosphorylation regulates nervous system development and function. Neuron 72:72-85. CrossRef Medline

Gräff J, Kim D, Dobbin MM, Tsai LH (2011) Epigenetic regulation of gene expression in physiological and pathological brain processes. Physiol Rev 91:603-649. CrossRef Medline

Guan JS, Haggarty SJ, Giacometti E, Dannenberg JH, Joseph N, Gao J, Nieland TJ, Zhou Y, Wang X, Mazitschek R, Bradner JE, DePinho RA, Jaenisch R, Tsai LH (2009) HDAC2 negatively regulates memory formation and synaptic plasticity. Nature 459:55-60. CrossRef Medline

Horch HW, Katz LC (2002) BDNF release from single cells elicits local dendritic growth in nearby neurons. Nat Neurosci 5:1177-1184. CrossRef Medline

Huang da W, Sherman BT, Lempicki RA (2009) Systematic and integrative analysis of large gene lists using DAVID bioinformatics resources. Nat Protoc 4:44-57. CrossRef Medline

Jakovcevski M, Akbarian S (2012) Epigenetic mechanisms in neurological disease. Nat Med 18:1194-1204. CrossRef Medline

Jan YN, Jan LY (2010) Branching out: mechanisms of dendritic arborization. Nat Rev Neurosci 11:316-328. CrossRef Medline

Jaworski J, Spangler S, Seeburg DP, Hoogenraad CC, Sheng M (2005) Control of dendritic arborization by the phosphoinositide-3'-kinase-Aktmammalian target of rapamycin pathway. J Neurosci 25:11300-11312. CrossRef Medline

Kim D, Frank CL, Dobbin MM, Tsunemoto RK, Tu W, Peng PL, Guan JS, Lee BH, Moy LY, Giusti P, Broodie N, Mazitschek R, Delalle I, Haggarty SJ, Neve RL, Lu Y, Tsai LH (2008) Deregulation of HDAC1 by p25/Cdk5 in neurotoxicity. Neuron 60:803-817. CrossRef Medline

Kim J, Daniel J, Espejo A, Lake A, Krishna M, Xia L, Zhang Y, Bedford MT (2006) Tudor, MBT and chromo domains gauge the degree of lysine methylation. EMBO Rep 7:397-403. CrossRef Medline

Kuppuswamy M, Vijayalingam S, Zhao LJ, Zhou Y, Subramanian T, Ryerse J, Chinnadurai G (2008) Role of the PLDLS-binding cleft region of CtBP1 in recruitment of core and auxiliary components of the corepressor complex. Mol Cell Biol 28:269-281. CrossRef Medline

Lahn BT, Page DC (1999) Retroposition of autosomal mRNA yielded testisspecific gene family on human Y chromosome. Nat Genet 21:429-433. CrossRef Medline

Lahn BT, Tang ZL, Zhou J, Barndt RJ, Parvinen M, Allis CD, Page DC (2002) Previously uncharacterized histone acetyltransferases implicated in mammalian spermatogenesis. Proc Natl Acad Sci U S A 99:8707-8712. CrossRef Medline

Li R, Zhang H, Yu W, Chen Y, Gui B, Liang J, Wang Y, Sun L, Yang X, Zhang Y, Shi L, Li Y, Shang Y (2009) ZIP: a novel transcription repressor, represses EGFR oncogene and suppresses breast carcinogenesis. EMBO J 28:2763-2776. CrossRef Medline

Li X, Liang J, Yu H, Su B, Xiao C, Shang Y, Wang W (2007) Functional consequences of new exon acquisition in mammalian chromodomain Y-like (CDYL) genes. Trends Genet 23:427-431. CrossRef Medline

Liu QR, Lu L, Zhu XG, Gong JP, Shaham Y, Uhl GR (2006) Rodent BDNF genes, novel promoters, novel splice variants, and regulation by cocaine. Brain Res 1067:1-12. CrossRef Medline

Lu B, Pang PT, Woo NH (2005) The yin and yang of neurotrophin action. Nat Rev Neurosci 6:603-614. CrossRef Medline

Ma DK, Marchetto MC, Guo JU, Ming GL, Gage FH, Song H (2010) Epigenetic choreographers of neurogenesis in the adult mammalian brain. Nat Neurosci 13:1338-1344. CrossRef Medline

Mei L, Xiong WC (2008) Neuregulin 1 in neural development, synaptic plasticity and schizophrenia. Nat Rev Neurosci 9:437-452. CrossRef Medline

Mortazavi A, Leeper Thompson EC, Garcia ST, Myers RM, Wold B (2006) Comparative genomics modeling of the NRSF/REST repressor network: from single conserved sites to genome-wide repertoire. Genome Res 16: 1208-1221. CrossRef Medline

Mulligan P, Westbrook TF, Ottinger M, Pavlova N, Chang B, Macia E, Shi YJ, Barretina J, Liu J, Howley PM, Elledge SJ, Shi Y (2008) CDYL bridges REST and histone methyltransferases for gene repression and suppression of cellular transformation. Mol Cell 32:718-726. CrossRef Medline

Nott A, Watson PM, Robinson JD, Crepaldi L, Riccio A (2008) S-Nitrosylation of histone deacetylase 2 induces chromatin remodelling in neurons. Nature 455: 411-415. CrossRef Medline

Park H, Poo MM (2013) Neurotrophin regulation of neural circuit development and function. Nat Rev Neurosci 14:7-23. CrossRef Medline

Parrish JZ, Kim MD, Jan LY, Jan YN (2006) Genome-wide analyses identify transcription factors required for proper morphogenesis of Drosophila sensory neuron dendrites. Genes Dev 20:820-835. CrossRef Medline

Parrish JZ, Emoto K, Jan LY, Jan YN (2007) Polycomb genes interact with the tumor suppressor genes hippo and warts in the maintenance of Drosophila sensory neuron dendrites. Genes Dev 21:956-972. CrossRef Medline

Peng YR, He S, Marie H, Zeng SY, Ma J, Tan ZJ, Lee SY, Malenka RC, Yu X (2009) Coordinated changes in dendritic arborization and synaptic strength during neural circuit development. Neuron 61:71-84. CrossRef Medline

Pruunsild P, Kazantseva A, Aid T, Palm K, Timmusk T (2007) Dissecting 
the human BDNF locus: bidirectional transcription, complex splicing, and multiple promoters. Genomics 90:397-406. CrossRef Medline

Qiu Z, Ghosh A (2008) A calcium-dependent switch in a CREST-BRG1 complex regulates activity-dependent gene expression. Neuron 60:775787. CrossRef Medline

Riccio A (2010) Dynamic epigenetic regulation in neurons: enzymes, stimuli and signaling pathways. Nat Neurosci 13:1330-1337. CrossRef Medline

Saito T, Nakatsuji N (2001) Efficient gene transfer into the embryonic mouse brain using in vivo electroporation. Dev Biol 240:237-246. CrossRef Medline

Sawarkar R, Paro R (2010) Interpretation of developmental signaling at chromatin: the Polycomb perspective. Dev Cell 19:651-661. CrossRef Medline

Shi Y, Sawada J, Sui G, Affar el B, Whetstine JR, Lan F, Ogawa H, Luke MP, Nakatani Y, Shi Y (2003) Coordinated histone modifications mediated by a CtBP co-repressor complex. Nature 422:735-738. CrossRef Medline

Sholl DA (1953) Dendritic organization in the neurons of the visual and motor cortices of the cat. J Anat 87:387-406. Medline

Sin WC, Haas K, Ruthazer ES, Cline HT (2002) Dendrite growth increased by visual activity requires NMDA receptor and Rho GTPases. Nature 419:475-480. CrossRef Medline

Sylwestrak E, Scheiffele P (2013) Neuroscience: Sculpting neuronal connectivity. Nature 503:42-43. CrossRef Medline

Tahiliani M, Mei P, Fang R, Leonor T, Rutenberg M, Shimizu F, Li J, Rao A, Shi Y (2007) The histone H3K4 demethylase SMCX links REST target genes to X-linked mental retardation. Nature 447:601-605. CrossRef Medline

Tea JS, Chihara T, Luo L (2010) Histone deacetylase Rpd3 regulates olfactory projection neuron dendrite targeting via the transcription factor Prospero. J Neurosci 30:9939-9946. CrossRef Medline

Thoenen H (1991) The changing scene of neurotrophic factors. Trends Neurosci 14:165-170. CrossRef Medline

Timmusk T, Palm K, Lendahl U, Metsis M (1999) Brain-derived neurotrophic factor expression in vivo is under the control of neuronrestrictive silencer element. J Biol Chem 274:1078-1084. CrossRef Medline
Tusher VG, Tibshirani R, Chu G (2001) Significance analysis of microarrays applied to the ionizing radiation response. Proc Natl Acad Sci U S A 98: 5116-5121. CrossRef Medline

Vermeulen M, Eberl HC, Matarese F, Marks H, Denissov S, Butter F, Lee KK, Olsen JV, Hyman AA, Stunnenberg HG, Mann M (2010) Quantitative interaction proteomics and genome-wide profiling of epigenetic histone marks and their readers. Cell 142:967-980. CrossRef Medline

Wan L, Hu XJ, Yan SX, Chen F, Cai B, Zhang XM, Wang T, Yu XB, Xiang AP, Li WQ (2013) Generation and neuronal differentiation of induced pluripotent stem cells in Cdyl-/- mice. Neuroreport 24:114-119. CrossRef Medline

Wang Y, Zhang H, Chen Y, Sun Y, Yang F, Yu W, Liang J, Sun L, Yang X, Shi L, Li R, Li Y, Zhang Y, Li Q, Yi X, Shang Y (2009) LSD1 is a subunit of the NuRD complex and targets the metastasis programs in breast cancer. Cell 138:660-672. CrossRef Medline

Wills ZP, Mandel-Brehm C, Mardinly AR, McCord AE, Giger RJ, Greenberg ME (2012) The nogo receptor family restricts synapse number in the developing hippocampus. Neuron 73:466-481. CrossRef Medline

Wong RO, Ghosh A (2002) Activity-dependent regulation of dendritic growth and patterning. Nat Rev Neurosci 3:803-812. CrossRef Medline

Wu H, Chen Y, Liang J, Shi B, Wu G, Zhang Y, Wang D, Li R, Yi X, Zhang H, Sun L, Shang Y (2005) Hypomethylation-linked activation of PAX2 mediates tamoxifen-stimulated endometrial carcinogenesis. Nature 438: 981-987. CrossRef Medline

Wu JI, Lessard J, Olave IA, Qiu Z, Ghosh A, Graef IA, Crabtree GR (2007) Regulation of dendritic development by neuron-specific chromatin remodeling complexes. Neuron 56:94-108. CrossRef Medline

Zhang Y, Yang X, Gui B, Xie G, Zhang D, Shang Y, Liang J (2011) Corepressor protein CDYL functions as a molecular bridge between polycomb repressor complex 2 and repressive chromatin mark trimethylated histone lysine 27. J Biol Chem 286:42414-42425. CrossRef Medline

Zuccato C, Tartari M, Crotti A, Goffredo D, Valenza M, Conti L, Cataudella T, Leavitt BR, Hayden MR, Timmusk T, Rigamonti D, Cattaneo E (2003) Huntingtin interacts with REST/NRSF to modulate the transcription of NRSE-controlled neuronal genes. Nat Genet 35:76-83. CrossRef Medline 